\title{
Industrial Photobioreactors and Scale-Up Concepts
}

\author{
Jeremy Pruvost*,1, Francois Le Borgne ${ }^{\dagger}$, Arnaud Artu $^{*, \dagger}$, \\ Jean-François Cornet ${ }^{*}$, Jack Legrand* \\ ${ }^{\star}$ GEPEA, Université de Nantes, CNRS, UMR6144, Bd de l'Université, Saint-Nazaire Cedex, France \\ †AlgoSource Technologies, Bd de l'Université, Saint-Nazaire Cedex, France \\ tUniversité Clermont Auvergne, ENSCCF, Clermont-Ferrand, France and CNRS, Institut Pascal, \\ Aubiere, France \\ ${ }^{1}$ Corresponding author: e-mail address: jeremy.pruvost@univ-nantes.fr
}

\section{Contents}

1. Introduction 258

2. PBR Engineering and Scaling Rules 259

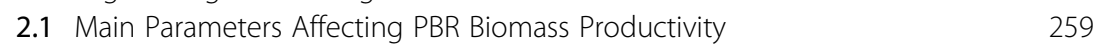

$\begin{array}{ll}\text { 3. Modeling PBRs } & 274\end{array}$

$\begin{array}{ll}3.1 \text { Introduction } & 274\end{array}$

3.2 Overview of Light-Limited Growth Modeling in a PBR 275

3.3 Kinetic Growth Model $\quad 276$

$\begin{array}{ll}\text { 3.4 Modeling of Radiative Transfer } & 279\end{array}$

$\begin{array}{ll}\text { 3.5 Determination of Radiative Properties } & 281\end{array}$

$\begin{array}{ll}3.6 \text { Solar PBR Modeling } & 281\end{array}$

4. Optimization of PBR Operation 283

4.1 Understanding Light-Limited Growth 283

4.2 Optimizing Light Attenuation Conditions for Maximal Biomass Productivities in PBRs

4.3 Optimizing Light Attenuation in Solar Cultivation 288

5. Development of Commercial Technologies Based on PBR Engineering Rules 291

5.1 Introduction 291

$\begin{array}{ll}5.2 \text { Artificial Light Culture Systems } & 292\end{array}$

$\begin{array}{ll}5.3 \text { Industrial Technologies } & 295\end{array}$

$\begin{array}{ll}5.4 \text { Solar Technologies } & 297\end{array}$

6. Conclusion 304

$\begin{array}{ll}\text { Acknowledgments } & 304\end{array}$

$\begin{array}{ll}\text { References } & 304\end{array}$

\section{Abstract}

Unlike other more classical bioprocesses for heterotrophic growth (typically yeasts and bacteria) where mixing tanks have standard geometries, microalgal culture has no single standard geometry. The main reason is the need for a light supply, which (1) has 
spurred various technologies designed to maximize light use and (2) greatly increases process complexity, as light is a complex parameter to handle. However, in-depth and long-term modeling efforts have now yielded engineering tools to design, optimize, and control photobioreactors in a predictive and rational way.

Here we discuss the parameters to consider when designing and operating microalgal cultivation systems and how appropriate engineering rules can support optimal system design and operation. Once the practical and economic constraints of the final application have been appropriately factored in, it becomes possible to set a rational design of effective technologies. This is illustrated later in this chapter in examples of successful developments, some of which are commercially available via AlgoSource Technologies. The examples chosen serve to highlight the many applications of photobioreactors from lab-scale fundamental studies to large solar industrial production, and to illustrate how a handful of engineering rules frame the various photobioreactor design options (artificial light or natural sunlight, external or internal lighting, high-cell-density culture, and more).

\section{INTRODUCTION}

Photosynthetic growth in standard autotrophic conditions is based on the assimilation, under illumination, of inorganic carbon and mineral nutrients dissolved in the medium. The cultivation of photosynthetic microorganisms thus requires:

- a light source (solar or artificial, with an appropriate light spectrum in the photosynthetically active radiation (PAR) range, typically $0.4-0.7 \mu \mathrm{m}$ ),

- an inorganic carbon source (such as dissolved $\mathrm{CO}_{2}$ ),

- mineral nutrients (major nutrients such as N, S, P sources; micronutrients like $\mathrm{Mg}, \mathrm{Ca}, \mathrm{Mn}, \mathrm{Cu}$, or $\mathrm{Fe}$; etc.), - set culture conditions ( $\mathrm{pH}$, temperature).

Ideally, the culture system has to enable optimal control of growth conditions, but it also has to meet the many and varied practical and economic tied to different microalgae applications, from small-scale lab production to mass-scale solar culture.

Generally speaking, microalgae cultivation shares many features with bioreactors in general, such as thermal regulation, nutrient feeding procedures, $\mathrm{pH}$ regulation, and mixing to enhance heat and mass transfers. However, the fact that photosynthetic growth needs a light supply has repercussions all the way from culture system design to effective operation (as detailed later in this chapter). An immediate observation is that, unlike other more classical bioprocesses where mixing tanks essentially have standard geometries, microalgal cultivation is characterized by a broad diversity 
of systems, ranging from open ponds (open systems) to photobioreactor (PBR) technologies (closed systems).

Detailed descriptions of existing geometries can be found in the literature (Carvalho et al, 2006; Lehr and Posten, 2009; Richmond, 2004b; Ugwu et al, 2008). The aim here is not to exhaustively review the different culture systems but to describe how system design and optimal operation can be encompassed in a robust and rational engineering approach. This will be illustrated by a handful of examples illustrating how factoring in the practical and economic constraints of the final application during the engineering phase ultimately results in very different technology designs from the same rational engineering tools. The focus will be on PBR technology as it offers the greatest potential in terms of optimization.

\section{PBR ENGINEERING AND SCALING RULES}

\subsection{Main Parameters Affecting PBR Biomass Productivity}

\subsubsection{Engineering Parameters}

Bioprocess design starts with identifying engineering parameters affecting process efficiency. This was the purpose of a research effort aiming to establish models able to represent microalgal biomass productivity in various PBR designs. The effort focused on addressing how to represent the influence of light supply on its use for photosynthetic growth in bulk culture (see later for a detailed example of a modeling approach that proved valid in several conditions). The work of Cornet and Dussap (2009) laid the foundations, as they developed an in-depth modeling approach for setting simple engineering rules able to predict maximal biomass productivities in cultivation systems. As maximal productivities are achieved when light only limits growth, engineering parameters related to light use were clarified. This was first published for constant artificial illumination conditions and then extended to the case of solar use by introducing specific features such as effect of the incident angle $\theta$ and the diffuse-direct distribution of solar radiation on resulting conversion in the cultivation system (Pruvost and Cornet, 2012). These relations give maximal surface $\left(S_{X \max }\right)$ and volumetric $\left(P_{X \max }\right)$ productivities:

$$
\begin{aligned}
\overline{S_{X \max }}= & \left(1-f_{\mathrm{d}}\right) \rho_{\mathrm{M}} \bar{\varphi}_{x} \frac{2 \alpha}{1+\alpha}\left[\frac{\bar{x}_{\mathrm{d}} K}{2} \ln \left[1+\frac{2 \bar{q}}{K}\right]\right. \\
& \left.+\left(1-\bar{x}_{\mathrm{d}}\right) \overline{\cos \theta} K \ln \left[1+\frac{\bar{q}}{K \overline{\cos \theta}}\right]\right]
\end{aligned}
$$


with

$$
\overline{P_{X \max }}=\overline{S_{X \max }} \frac{S_{\text {light }}}{V_{\mathrm{R}}}=\overline{S_{X \max }} a_{\text {light }}
$$

where - denotes a time averaging, ie, quantities averaged over a given period of exploitation. Averaging is typically applied in solar conditions due to the variation in irradiation conditions, leading to average performances on representative periods of exploitation (ie, $24 \mathrm{~h}$, month, season, year, etc.).

The parameters of Eq. (1) can be split into three groups:

- Parameters related to the cultivated species: mean mass quantum yield $\bar{\varphi}_{X}^{\prime}$, half-saturation constant for photosynthesis $K$, and linear scattering modulus $\alpha$ related to the microorganism's radiative properties (see Table 1 for an example of parameters for Chlorella vulgaris).

- Parameters related to the operating conditions: incident angle $\theta$, total collected photon flux density (ie, PFD) $\bar{q}$, and corresponding diffuse fraction $\bar{x}_{\mathrm{d}}$ (here averaged over the period of exploitation).

- Parameters related to PBR geometry: specific illuminated area $a_{\text {light }}$ given by ratio of PBR illuminated area to total culture volume, design dark volume fraction $f_{\mathrm{d}}$ which represents any volume fraction of the PBR not lit by incident PFD (eg, nonlit mixing tank).

Table 1 Examples of Growth Model Parameters for Chlorella vulgaris (Values Are Given for Growth on Ammonia as N-Source)

\begin{tabular}{lcc} 
Parameter & Value & Unit \\
\hline$\rho_{\mathrm{M}}$ & 0.8 & - \\
$J_{\mathrm{NADH}_{2}}$ & $1.8 \times 10^{-3}$ & $\mathrm{~mol}_{\mathrm{NADH}_{2}} \mathrm{~kg}_{X}^{-1} \mathrm{~s}^{-1}$ \\
$v_{\mathrm{O}_{2}-X}$ & 1.13 & - \\
$\bar{\varphi}_{\mathrm{O}_{2}}^{\prime}$ & $1.1 \times 10^{-7}$ & $\mathrm{~mol}_{\mathrm{O}_{2}} \mu \mathrm{mol}_{h \nu}^{-1}$ \\
$\bar{\varphi}_{X}$ & $2.34 \times 10^{-9}$ & $\mathrm{~kg}_{X} \mu \mathrm{mol}_{h \nu}^{-1}$ \\
$M_{X}$ & 0.024 & $\mathrm{~kg}_{X} \mathrm{C}-\mathrm{mol}^{-1}$ \\
$v_{\mathrm{NADH}}-\mathrm{O}_{2}$ & 2 & - \\
$K_{A}$ & 30,000 & $\mu \mathrm{mol}_{h \nu} \mathrm{kg}^{-1} \mathrm{~s}^{-1}$ \\
$K$ & 110 & $\mu \mathrm{mol}_{h \nu} \mathrm{m}^{-2} \mathrm{~s}^{-1}$ \\
$K_{r}$ & 150 & $\mu \mathrm{mol}_{h \nu} \mathrm{kg}^{-1} \mathrm{~s}^{-1}$ \\
$A_{c}$ & 1500 & $\mu \mathrm{mol}_{h \nu} \mathrm{kg}^{-1} \mathrm{~s}^{-1}$ \\
$E a$ & 270 & $\mathrm{~m}^{2} \mathrm{~kg}^{-1}$ \\
$E s$ & 2780 & $\mathrm{~m}^{2} \mathrm{~kg}^{-1}$ \\
$b$ & 0.002 & - \\
\hline
\end{tabular}


For a given species, parameters affecting PBR productivity are designspecific illuminated area $a_{\text {light }}$, design dark fraction of the reactor $f_{\mathrm{d}}$, and ability of the PBR to collect light (characterized by incident PFD $\bar{q}$ and related incident angle $\theta$ and diffuse fraction $\bar{x}_{\mathrm{d}}$ ). All these parameters are tied to light supply. Light collected by the PBR is obviously a function of its location and weather conditions.

These engineering formulae can be simplified, especially in the case of artificial light. In artificial light, the light source is often set to provide normal incidence $(\overline{\cos \theta}=1)$, as this also corresponds to a maximization of the light provided to the culture. It is also common practice to apply quasi-collimated light $\left(\bar{x}_{\mathrm{d}}=0\right)$ as obtained from LED panels (ie, without combination to diffuse plate). This leads to the following simplified formula:

$$
\overline{S_{X \max }}=\left(1-f_{\mathrm{d}}\right) \rho_{\mathrm{M}} \bar{\varphi}_{X} \frac{2 \alpha}{1+\alpha} K \ln \left[1+\frac{\bar{q}}{K}\right]
$$

with

$$
\overline{P_{X \max }}=a_{\text {light }}\left(1-f_{\mathrm{d}}\right) \rho_{\mathrm{M}} \bar{\varphi}_{X} \frac{2 \alpha}{1+\alpha} K \ln \left[1+\frac{\bar{q}}{K}\right]
$$

Obviously, these formulae only provide maximal performances which, as explained later, can only be achieved if other conditions are fulfilled, especially in actual culture system operating. It also assumes that light alone limits growth, assuming all other biological needs (nutrients, dissolved carbon) and operating conditions ( $\mathrm{pH}$, temperature) are controlled at optimal values (Cornet, 2010; Pruvost and Cornet, 2012; Takache et al, 2010). In practice, this could prove a big challenge (especially in mass-scale outdoor production, see later), but the relations that can be easily used (ie, analytic formulae) already give highly valuable information in the preliminary engineering phase.

These relations also underline the relevant engineering parameters affecting PBR productivity, ie, specific illuminated area $a_{\text {light }}$, engineering dark fraction $f_{\mathrm{d}}$, and light collected $\bar{q}$. Note that dark fraction must not be confused with dark volume which results from light attenuation in the culture volume due to light absorption by photosynthetic cells (see later). This reflects to unlit fractions of the culture system, resulting from the design itself, and is typically obtained when adding a dark tank in the hydraulic loop for cooling or $\mathrm{pH}$ regulation purposes for example, or when a nonilluminated airlift vertical tube is introduced for culture mixing and circulation. To maximize PBR performance, the design dark fraction should 
be minimized down to negligible or null values, but this condition is not always met in practice.

Fig. 1 shows the influence of these engineering parameters on maximal productivities as predicted by these relations, here for the case of C. vulgaris
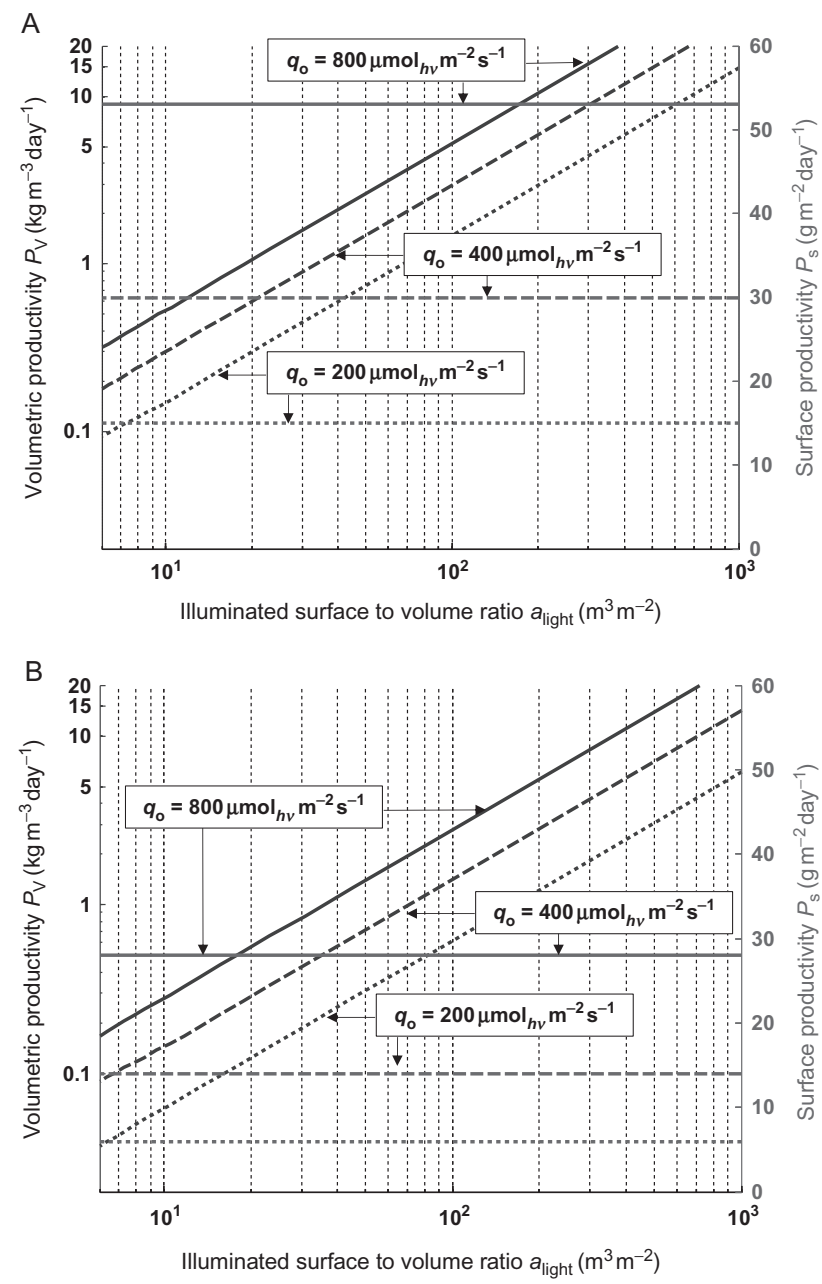

Figure 1 Influence of the illuminated surface to volume ratio $\left(a_{\text {light }}\right)$ on PBR productivities. A direct influence on volumetric productivity is shown (two orders of magnitude of variation). Surface productivity is found independent of this engineering parameter. PFD $\left(q_{0}\right)$ reveals to have a positive effect on both values. Influence of the design dark volume fraction of the PBR $\left(f_{\mathrm{d}}\right)$ is also illustrated. Panel $(\mathrm{A})$ is the best design case, namely without design dark volume fraction $\left(f_{d}=0\right)$, and panel (B) is for a PBR design presenting $20 \%$ of its total volume in the dark $\left(f_{\mathrm{d}}=0.2\right)$. Results are given for $C$. vulgaris, and all values correspond to maximal performances (ie, as obtained in continuous cultivation, light-limited conditions, luminostat " $\gamma=1$ " regime). 
cultivation. In addition to the ideal condition of no dark fraction in the cultivation system $\left(f_{\mathrm{d}}=0\right.$, Fig. $\left.1 \mathrm{~A}\right)$, a typical dark fraction value of $20 \%$ was also considered $\left(f_{\mathrm{d}}=0.2\right.$, Fig. $\left.1 \mathrm{~B}\right)$. The figures illustrate the main guiding rules of PBR engineering:

- Specific illuminated surface $a_{\text {light }}$ has a huge influence on volumetric productivity (two orders of magnitude are covered here) but no influence on surface productivity. Indeed, it is well known that productivity, when expressed per unit surface area and under light limitation, is independent of PBR depth as it is only dependent on the light collected in lightlimited growth conditions, which is defined by the PBR collecting surface and not its volume (Cornet, 2010; Lee et al, 2014).

- PFD $\bar{q}$ is a relevant parameter as it has a positive effect on both surface and volumetric productivities. In solar conditions, the PFD will be defined by the ability of the system to collect light, which will depend on PBR geometry, geographical location, and positioning, as shown in numerous works (Acién Fernández et al, 2001; Chen et al, 2006; Chini Zittelli et al, 2000; Doucha and Livansky, 2006; Molina et al, 2001; Oswald, 1988; Pruvost, 2011; Pruvost and Cornet, 2012; Pruvost et al, 2012; Richmond and Cheng-Wu, 2001).

- The design dark volume fraction $f_{\mathrm{d}}$ has a highly negative influence on both surface and volumetric productivities. This is especially the case for microalgae presenting significant respiration activity in the dark. The dark volume fraction is not only a nonproducing volume but also contributes negatively to the overall PBR performance due to biomass catabolism in this nonilluminated volume. As a result, a dark volume fraction of $20 \%$ can decrease PBR productivities by a factor of 2 for $C$. vulgaris. Note that dark volume is usually introduced in design practice for microalgal cultivation units (ie, mixing tank in the cultivation loop of a tubular system, nonilluminated volume of an airlift PBR, etc.).

Results of Fig. 1 also show that due to the progressive saturation of photosynthetic conversion (as represented by parameter $K$, which is species dependent), an increase of PFD received on the cultivation system will increase productivity (as shown in Fig. 1) but will also decrease the thermodynamic efficiency of the process (ie, yield of conversion of light energy into biomass). This is shown in Fig. 1 by the values of surface productivities obtained for different PFDs. Increasing the PFD from 400 to $800 \mu \mathrm{mol}_{h \nu} \mathrm{m}^{-2} \mathrm{~s}^{-1}$ (a 2-fold increase) leads to an increase in surface productivity from 30 to $52 \mathrm{~g} \mathrm{~m}^{-2} \mathrm{day}^{-1}$ (a 1.7 -fold increase).

This highlights the importance of the light dilution principle, as obtained from insertion of light sources inside the culture volume (leading to what are 
dubbed "volumetrically lightened" systems; see Cornet, 2010), wherein the surface illuminating the culture becomes higher than the surface directly exposed to the light source (light capture surface; see Fig. 2). This results in light dilution which increases light conversion yield by photosynthesis. When expressed per unit of light capture surface, biomass productivity is higher with volumetrically lightened systems than surface-lightened systems, but these technologies carry several drawbacks, including higher technological complexity (need for optical capture devices), which can inflate costs, and the fact that efficiency hinges on proper design. The main challenges are to deliver a light flux at the required value for optimal photosynthetic conversion by the culture, and the need to engineer an optical capture device that minimizes loss of light energy when transmitting light from capture to culture. Diluting light also entails a trade-off with volumetric productivity (ie, biomass concentration) and will thus have to be compensated to a certain extent by an increase of specific illuminated surface. This leads to specific technologies such as the DiCoFluV concept, which will be detailed further (Cornet, 2010). Despite the challenges of setting these culture systems, the effort can pay off, especially in the case of solar production, where biomass productivity per unit of land area (ie, capture surface) could be a relevant factor.

\subsubsection{Operating Parameters}

Light, carbon and mineral nutrient supply, temperature, and $\mathrm{pH}$ are the main variables liable to limit photosynthetic growth and thus reduce the productivity of cultivation systems (assuming there is no predatory contamination). Except for light, these parameters can be controlled and set at optimal or near-optimal values with appropriate engineering and operating procedures. This is where PBRs, as a closed geometry, have a critical advantage, although even here the engineering of the culture system, eg, thermal regulation or carbon supply, still proves highly influential.

\subsubsection{Thermal Regulation}

Like in any biological process, temperature directly influences photosynthesis and microorganism growth. Particularly under solar illumination, closed-system PBRs tend to overheat whereas open-system PBRs can suffer evaporation of water under strong incident irradiance, explained by culture confinement and the strongly exoenergetic photosynthetic growth (Carvalho et al, 2011; Hindersin et al, 2013; Torzillo et al, 1996; Wilhelm and Selmar, 2011). In fact, thermodynamic efficiency over the 


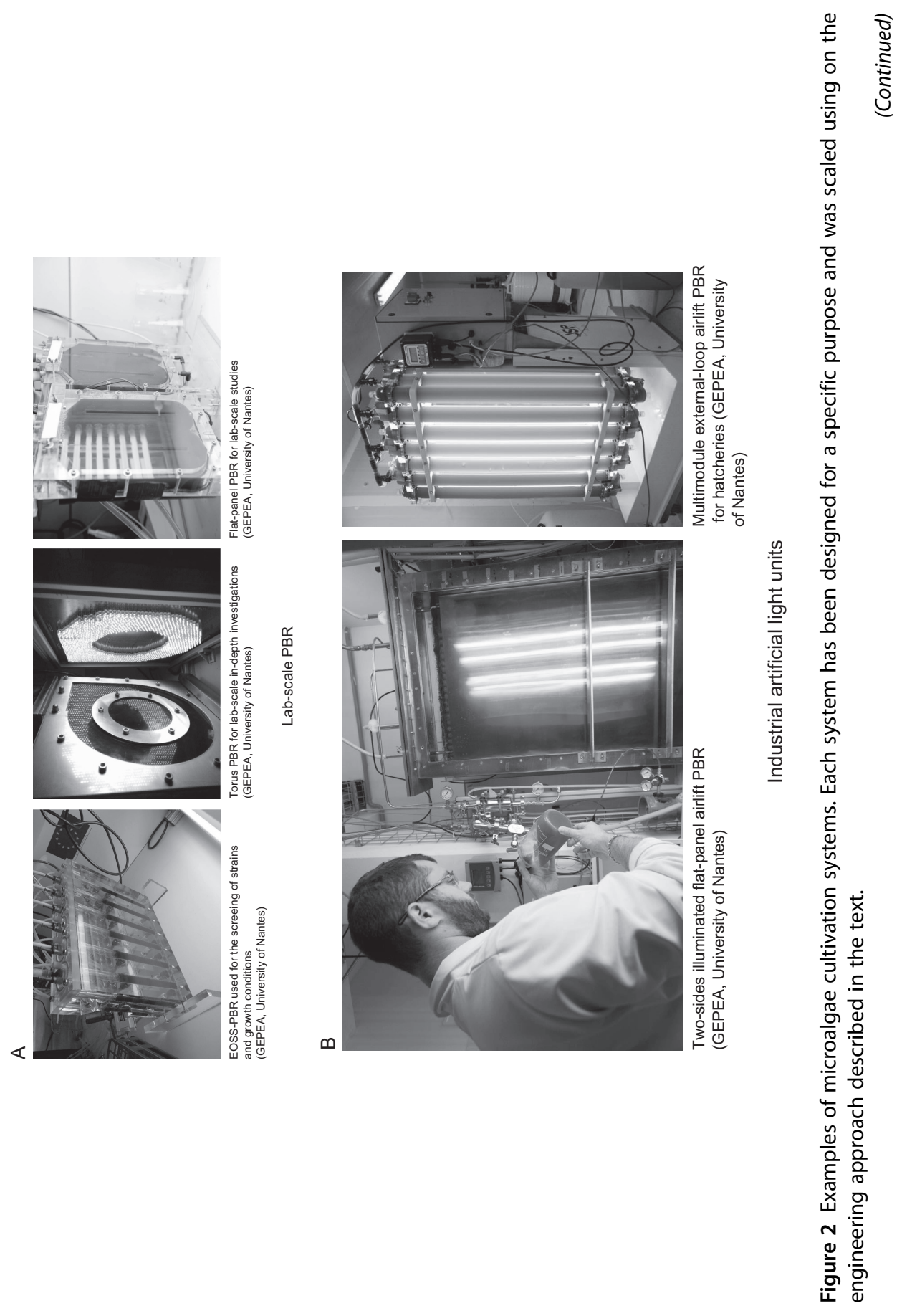



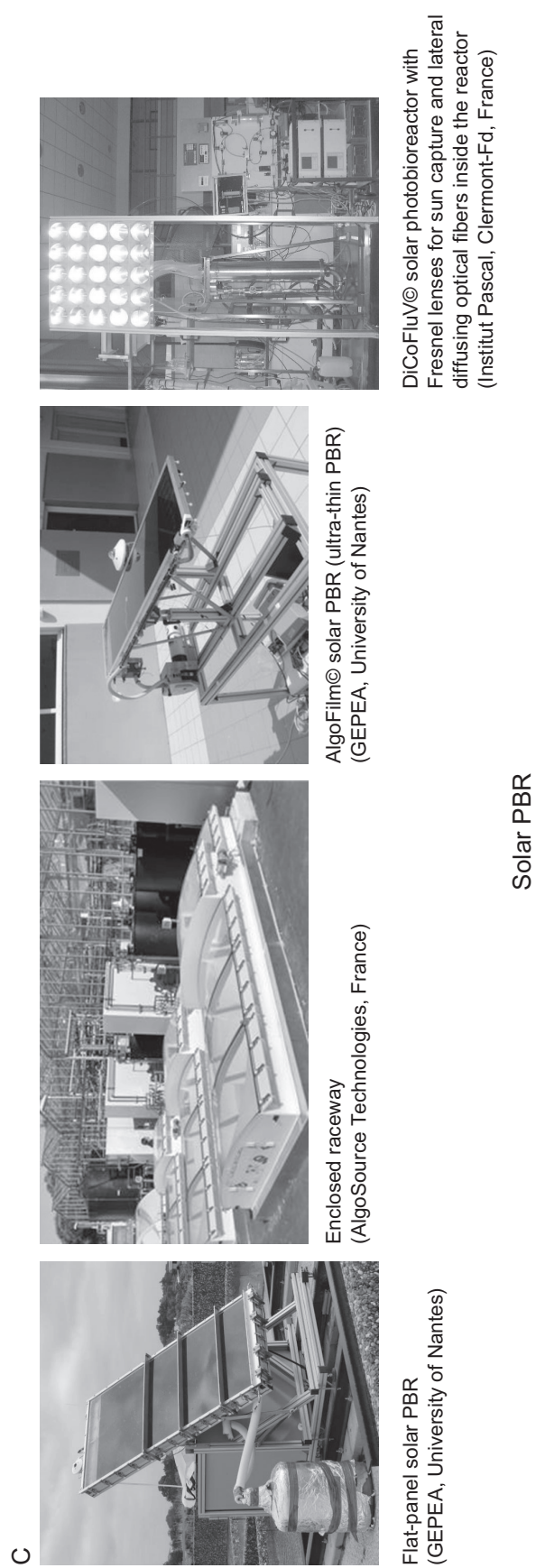

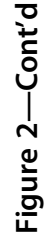


PAR region of systems working with low light typical of artificial illumination $\left(100-300 \mu \mathrm{mol}_{h \nu} \mathrm{m}^{-2} \mathrm{~s}^{-1}\right)$ is generally below 5\% (Cornet, 2010) and decreases to $2 \%$ under large solar irradiance $\left(>500 \mu \mathrm{mol}_{h \nu} \mathrm{g}^{-2} \mathrm{~s}^{-1}\right)$. As a result, around $95 \%$ of the captured light is converted into heat by biochemical reactions and dissipation in light-collecting antennas. In fact, under outdoor conditions, around $50 \%$ of the energy in the solar radiation is contained in the near- and mid-infrared above $750 \mathrm{~nm}$ and directly participates in heating up the culture (Goetz et al, 2011; Hindersin, 2013; Hindersin et al, 2013, 2014).

Thermal regulation of PBRs has been widely investigated as a major issue of solar microalgal cultivation (Borowitzka, 1999; Grobbelaar, 2008; Hindersin et al, 2013, 2014). Unfortunately, without proper thermoregulation, temperatures lethal to living microorganisms can easily be reached inside the solar PBR, illustrating why PBR cooling is a usually a major engineering issue. In winter in temperate climates, excessively low temperatures can result in loss of biomass growth and productivity, so heating the culture can be beneficial (Hindersin, 2013). The appropriate temperature window is strongly dependent on species cultivated but typically in the $10-30^{\circ} \mathrm{C}$ range.

Various solutions are available for heating or cooling PBRs depending on PBR technology, size, and location. Water cooling and/or heating by spraying on the outside PBR surfaces or by direct PBR immersion in a pool are often used (Borowitzka, 1999). In temperate regions, cultivation systems can also be placed in greenhouses. Although efficient, these methods can increase the build and operating costs and negatively impact environmental footprint through excessive energy and water use.

Although technical solutions exist, PBR temperature control remains a challenge under solar conditions, especially if the design brief is for a costeffective solution offering low-energy consumption and year-round operation which may need both cooling and heating. The engineering of the cultivation system is also relevant. Goetz et al (2011) experimentally and theoretically investigated the effect of various flat-panel PBR designs and found a decrease of up to one order of magnitude in PBR energy consumption depending on configuration. IR filtering, for example, was found to be especially effective at reducing culture overheat. More recently, research efforts have investigated the integration of PBR technology in building façades. This integration offers various benefits in terms of thermal management of both PBRs and buildings. Energy exchanges between the building and the PBRs can be designed so as to cool or warm each subsystem. For example, PBRs can filter sunlight in summer to reduce the thermal load 
on the building. In winter, excess thermal energy in the cultivation system can be used to warm the building. Note also that the added thermal mass of the building can be used to facilitate PBR thermal regulation regardless of season.

Overall, PBR thermal regulation depends on location, time of year, and strain cultivated. Cooling and/or heating requirements have to be estimated (usually in the range $50-200 \mathrm{~W} / \mathrm{m}^{2}$ ) and the associated thermal solutions should be defined and integrated well upstream in system design. For climates with large variations in outdoor temperature and solar irradiation over the course of a year, it could be beneficial to cultivate different species with optimal growths at different temperatures (Hindersin, 2013), which could significantly decrease energy needs over the period of exploitation.

\subsubsection{Carbon and Mineral Nutrient Requirements}

Assuming the growth medium of the strain is known, growth limitation by mineral nutrients can easily be avoided. The growth medium has to contain all the necessary (macro and micro) nutrients in sufficient quantities and must therefore be adjusted according to the biomass concentration planned. Stoichiometric growth equations can be used for this purpose or, more simply, concentrations can be monitored during cultivation. In specific cases, it would also be of interest to apply mineral limitation to induce specific metabolic responses, such as lipid accumulation ( $\mathrm{N}$ source deprivation) or hydrogen production (sulfur deprivation). This is where combining mass balances on the cultivation system with stoichiometric growth equations is useful. Interested readers can refer to studies in which the method has been applied to various species (Degrenne et al, 2011b; Pruvost, 2011; Pruvost et al, 2009).

The inorganic carbon source should ideally be $\mathrm{CO}_{2}$ dissolved in the culture medium, which makes preventing growth limitation by the carbon source more problematic. This depends on dissolved carbon concentration and thus on the gas-liquid mass transfer rate in the PBR. $\mathrm{CO}_{2}$ dissolution also affects $\mathrm{pH}$ value (acidification), which in turn influences the amount and form of dissolved carbon obtained $\left(\mathrm{CO}_{2}, \mathrm{HCO}_{3}{ }^{-}\right.$, or $\left.\mathrm{CO}_{3}{ }^{2-}\right)$. Nutrient consumption can also interact with $\mathrm{pH}$ evolution (especially nitrogen source due to its significant consumption). Keeping an optimal $\mathrm{pH}$ value for growth while averting limitation by the carbon source may thus prove challenging. In most cases, simple $\mathrm{CO}_{2}$ bubbling is found to suffice in the first instance for both $\mathrm{pH}$ regulation (acidification) and carbon feeding, but specific cases, such as when using an ammonium source (the consumption of which also 
leads to acidification), could present a more difficult challenge. Dissolved carbon concentration can always be monitored experimentally to forestall limitation (Degrenne et al, 2010; Le Gouic, 2013).

\subsubsection{3 pH Control}

Photoautotrophic microorganisms are cultivated in an aqueous solution in which the inorganic carbonaceous substrate is supplied through the dissolution of $\mathrm{CO}_{2}$ gas in water or through the speciation of carbonates from the culture media. In most cases, the $\mathrm{CO}_{2}$ is supplied in the form of fine bubbles. In water, the $\mathrm{CO}_{2}$ gas forms other species such as dissolved carbon dioxide $\left(\mathrm{CO}_{2 \mathrm{aq}}\right)$, carbonic acid $\left(\mathrm{H}_{2} \mathrm{CO}_{3}{ }^{-}\right)$, bicarbonate $\left(\mathrm{HCO}_{3}{ }^{-}\right)$, and carbonate $\left(\mathrm{CO}_{3}{ }^{2-}\right)$, whose sum is termed TIC (total inorganic carbon). Many species of microalgae have developed mechanisms that enable both $\mathrm{CO}_{2 \mathrm{aq}}$ and $\mathrm{HCO}_{3}{ }^{-}$to support photosynthesis, but $\mathrm{CO}_{2 \mathrm{aq}}$ is still required. It is obtained by splitting the bicarbonate inside the cell $\left(\mathrm{HCO}_{3}{ }^{-} \leftrightarrow \mathrm{CO}_{2 \mathrm{aq}}+\mathrm{OH}^{-}\right)$, a reaction that releases hydroxyl ions, causing the increase in $\mathrm{pH}$. The ratio of $\mathrm{CO}_{2 a q}$ to $\mathrm{HCO}_{3}{ }^{-}$depends closely on $\mathrm{pH}$, as bicarbonate is the dominant species in solutions of $\mathrm{pH}>6.3$, and the conversion of $\mathrm{HCO}_{3}{ }^{-}$to $\mathrm{CO}_{2 \mathrm{aq}}$ is very fast. Thus, when $\mathrm{CO}_{2 \mathrm{aq}}$ is removed from the medium, $\mathrm{pH}$ will increase. Microalgal cultivation often entails $\mathrm{pH}$ control by means of $\mathrm{CO}_{2}$ gas bubbled into the reactor. This fresh supply of $\mathrm{CO}_{2}$ will shift the equilibrium by lowering the $\mathrm{pH}$. Ifrim et al (2014) proposed a global photoautotrophic growth model in which a radiative transfer model, a biological model, and a thermodynamic model are coupled. This model can accurately predict the dynamics of $\mathrm{pH}$ evolution.

\subsubsection{Transfer Phenomena}

Fluid dynamics in PBRs is import on several fronts. Although many studies have shown the relevance of mixing conditions in microalgal cultivation systems, there is still insufficient knowledge to provide engineering rules for their systematic optimization. Hydrodynamic conditions can have several outcomes, some of which are common to other bioprocesses (hydrodynamic shear stress, mass and heat transfer enhancement, cell sedimentation, and biofilm formation) while others are specific to microalgal cultivation systems, and especially for light-dark (L/D) cycle effects. L/D cycles result from cell displacement in the heterogeneous radiation field, such that cells experience a specific history with respect to the light they absorb, composed of variations from high irradiance level (in the vicinity of the light source) to low or quasi-nil values (deep in the culture) if biomass concentration is high. 
As widely described in the literature (Janssen et al, 2000; Perner-Nochta and Posten, 2007; Pruvost et al, 2008; Richmond, 2004a; Rosello Sastre et al, 2007), this dynamic fluctuating regime can influence photosynthetic growth and thereby process efficiency. Note, however, that hydrodynamic timescales are several orders of magnitude greater than photosynthesis timescales, so the effects of $\mathrm{L} / \mathrm{D}$ cycles due to hydrodynamics can in most cases be considered negligible (Pruvost et al, 2008), which is not the case for the presence of dark zones, as shown later (Section 3).

PBRs are generally considered perfect mixing systems, with homogenized nutrient concentrations and uniform biomass concentrations. An important point is to reduce the energy consumption for mixing by maintaining efficient mixing, which is contingent on the type of PBR. Numerical simulations could be one way to optimize flow configuration and mixing, including characterization of light regimes in cultivation systems by a Lagrangian simulation (Pruvost et al, 2002a, 2002b). CFD can be used to gain an in-depth understanding of the hydrodynamics/flow pattern in the PBRs and usefully inform scale-up. For bubble-flow PBRs, most published simulations have used two-phase models (air and water) and employed the Eulerian-Eulerian mixture model (Bitog et al, 2011). To increase radial mixing in flat-panel airlift systems, static mixers can be used (Subitec PBR) to direct the flow toward the light source (Bergmann et al, 2013). For stirred PBRs, the choice of impeller type is important (Pruvost et al, 2006). If species cultivated are not stress sensitive, the more efficient flow circulation and mixing impeller could be used. If not the case, a compromise must be found based on the strain's sensitivity to shear stress. Numerical simulation of the flow system can offer the ability to design a raceway before construction, saving considerable cost and time. Moreover, the impacts of various parameters, such as culture media depth, temperature, flow speeds, baffles, could be investigated to optimize operating conditions (James et al., 2013).

$\mathrm{CO}_{2}$ mass transfer is one of the more important transfer phenomena issues in PBRs. $\mathrm{CO}_{2}$ is the usual carbon source for photosynthetic culture of microalgae and is generally supplied by continuous or intermittent gas injection. As the carbon is consumed, oxygen is ultimately produced by photolysis of water and released into the culture medium, where it can be removed by gas stripping. Volumetric gas-liquid mass transfer, $k_{\mathrm{La}}$, is related to power input per volume due to aeration (Acién et al., 2012; Chisti, 1989). The volumetric gas-liquid mass transfers for oxygen and for $\mathrm{CO}_{2}$ are related to their diffusion coefficients in the culture media. 


\subsubsection{Residence Time and Light Attenuation Conditions}

Biomass concentration has a critical influence as it directly impacts light attenuation regime in the culture volume. It can be controlled via the harvesting strategy. When operated in batch mode, the harvesting strategy consists of defining culture growth duration. For practical reasons, many mass-scale solar PBRs are operated either in batch mode with biomass harvesting at the end of the culture or in semi-continuous mode with spot harvesting of part of the culture and replacement by fresh growth medium. This means biomass concentration and thus light attenuation conditions evolve with time. In continuous mode, a steady state is achieved only if all operating parameters are maintained constant with time. This condition can be met in permanent illumination conditions (artificial light). The PBR is then operated with a constant permanent value of the residence time $\tau$ (or dilution rate $D=1 / \tau$ ), leading to a steady state with constant biomass concentration and light attenuation conditions.

Fig. 3A shows the strong relation between harvesting strategy (here defined by the residence time value), biomass concentration, light attenuation regime (here represented by the illuminated fraction $\gamma$; see Section 3.4), and resulting biomass productivity, as illustrated in the case of continuous culture. On one hand, if biomass concentration is too low (ie, low residence time), part of the incoming photons is not absorbed and is instead transmitted through the culture. This results in a loss of biomass productivity. In addition, light received per cell is high and may lead to further decreases in productivity due to increased photosynthetic dissipation. It may also induce cell photoacclimation resulting in a decrease in algal pigment content, leading to a higher light penetration with then further increase of the light received per cell. The system consequently becomes highly unstable, usually resulting in culture washout. Such low light attenuation conditions should thus be avoided in microalgal cultivation, especially for high PFDs typically larger than $200 \mu \mathrm{mol}_{h \nu} \mathrm{m}^{-2} \mathrm{~s}^{-1}$.

On the other hand, if biomass concentration is too high (ie, high residence time), a dark zone appears inside the culture. This dark zone is the direct consequence of light extinction by cells in suspension, whose effect can be positive in high-illumination conditions by reducing photoinhibition effects and thus increasing process stability (Carvalho et al, 2011; Grima et al, 1999; Richmond, 2004b). Note that for microorganisms like eukaryotic microalgae that show respiration activity under illumination, a dark zone in the culture volume promotes respiration, resulting in a loss of biomass productivity. Therefore, achieving the maximum biomass productivity in 

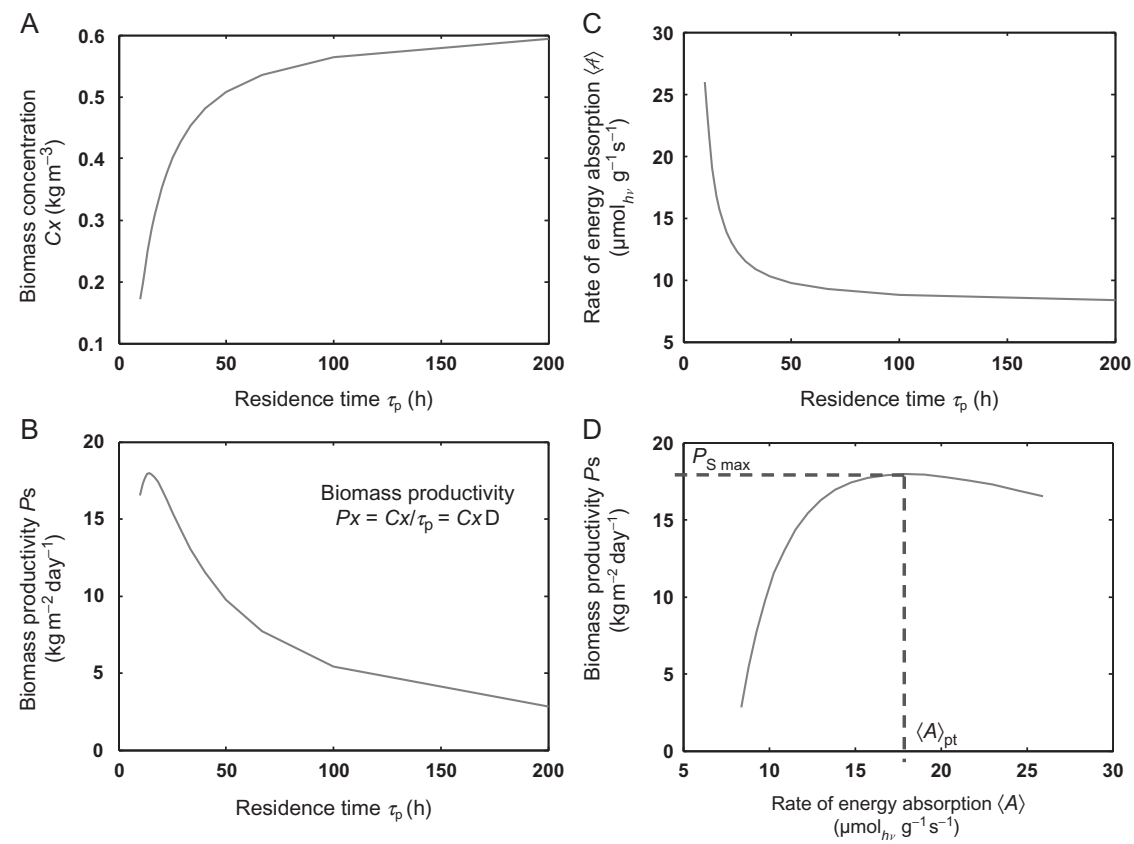

Figure 3 Evolution of biomass concentration (A), biomass productivity (B), and photon absorption rate $(C)$ as a function of the residence time applied to the cultivation system. This illustrates the strong relation between all variables in microalgal cultivation system, as explained by the direct effect on light attenuation conditions. The example is here given for $C$. vulgaris. (D) The relation between biomass productivity and photon absorption rate.

this case requires the exact condition of complete absorption of the incident light (Takache et al, 2010) but without a dark zone in the culture volume. This condition is often referred to as luminostat mode. Note that it should not be confused with turbidostat mode, which refers to a turbidity-based regulation of a continuous culture. This condition has also been introduced as the " $\gamma=1$ " condition, $\gamma$ denoting the ratio between illuminated volume and total cultivation system volume (see Section 3.4). For microorganisms with negligible respiration activity under illumination, such as prokaryotic cyanobacteria cells, fulfilling the condition of complete light absorption $(\gamma \leq 1)$ will be enough to reach maximum biomass productivity.

\subsubsection{Specific Rate of Photon Absorption A}

Another way to represent the strong correlation between light attenuation conditions and the associated biomass productivity is to calculate the specific rate of photon absorption, noted $A$ (here expressed per unit of biomass, ie, in 
$\left.\mu \mathrm{mol}_{h \nu} \mathrm{s}^{-1} \mathrm{~kg}^{-1}\right)$. Surprisingly, even though this value has been found beneficial in numerous studies devoted to photoreaction, it is rarely used in microalgal culture (Cassano et al, 1995). $A$ is obtained by integrating the product of spectral values of local irradiance $G_{\lambda}$ (see Section 3.4) and microalgae mass absorption coefficient $E a_{\lambda}$ (see Section 3.5) on the PAR region (Aiba, 1982; Cassano et al, 1995; Kandilian et al, 2013):

$$
A=\int_{\mathrm{PAR}} E a_{\lambda} G_{\lambda} \mathrm{d} \lambda
$$

This value have been demonstrated as useful in photoreactor or PBR modeling (Dauchet, 2012; Kandilian et al, 2014; Pruvost and Cornet, 2012), to relate light absorption conditions to (biological) reactions. The rate of photon absorption represents the light effectively absorbed by the cells, which is a combination of light received (irradiance $G$ ) and the ability of the cells to absorb light (absorption coefficient $\mathrm{Ea}$ ). As light absorption by cells depends of their pigment content, which is highly variable, rate of photon absorption was found more representative (both for kinetic modeling and cells regulation mechanisms) than considering the irradiance value alone.

Kandilian et al (2013) have shown the direct relation of the specific rate of photon absorption with lipid accumulation in the condition of nitrogen starvation, which is known to trigger lipid reserve (ie, TAG) accumulation but also to strongly decrease pigment content, thus altering light absorption by cells. The authors found that a given value of specific rate of photon absorption $A$ was necessary to trigger TAG overaccumulation, and also that TAG synthesis rate was strongly related to $A$.

More recently, Soulies et al (accepted) investigated the influence of specific lighting conditions such as a change in light spectrum or incident angle. Introducing specific rate of photon absorption $A$ was again found useful to relate these conditions to growth kinetics and thus make it possible to capture the respective influences of absorption rates and growth of red and white lights and non-normal incident angles. A key finding here was that white light decreases the negative effect of dark volumes. In contrast to red light, whose wavelengths were almost uniformly and rapidly absorbed in the culture volume, a part of the white light spectrum (ie, green light) was found to penetrate deeper in the culture volume meaning that at similar biomass concentration, white light showed a higher rate of absorption in the culture depth than red light. The net result was that this tended to decrease 
the expected positive effect of red light on biomass productivity. Those authors also reported marked pigment acclimation in the studied strain (ie, C. vulgaris) which tended to compensate the fast decrease in available light with culture depth (in the case of red light) but also non-normal incident angle. The rate of photon absorption was found to help efficiently represent all effects, and was then proven as a value of interest in microalgal culture optimization.

Generally speaking, introducing specific rate of photon absorption $A$ could find applications for any case where light absorption rates are potentially relevant. This could be in the optimization of light attenuation conditions for achieving maximal biomass productivity, but also in solar operations where light conditions tend to be oversaturating, leading to possible photoinhibition. These features are introduced in a typical example given in Fig. 3B. Increasing biomass concentration in the cultivation system will decrease the rate of photon absorption due to stronger light attenuation, thus resulting in smaller irradiance $G$. As a result, peak biomass productivity will be obtained at an optimal photon absorption rate value. For the case simulated in Fig. 3, this optimal value is typically situated around 15-

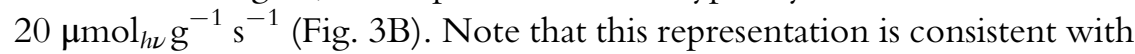
the condition of luminostat regime $(\gamma=1)$, and both approaches can be used to maximize the biomass productivity of any cultivation system.

\section{MODELING PBRs}

\subsection{Introduction}

The previous engineering rules (Eq. 1) make it possible to calculate the maximal performances of a given culture system as a function of design, light received, and cultivated strain. Such information is highly valuable for scaling the system as a function of operational constraints, ie, objective of biomass production, algae farming resources available (land area, irradiation conditions, etc.). In many cases, this information is considered sufficient for the engineer to estimate, for example, the number/size of production units and the allied capital and operational costs (ie, CAPEX and OPEX). Bear in mind that these relations give theoretical maximal productivities whereas, in practice, productivities will be lower for many reasons: nonideal culture conditions (temperature or $\mathrm{pH}$, dissolved carbon or medium, contamination), the strong influence of daytime culture conditions variation on growth kinetics (ie, weather conditions, day-night cycles), partial shading by other units or surrounding buildings or trees, nonoptimized harvesting 
strategies, and poor control of the irradiation field leading, for example, to photoinhibition phenomena.

The following section provides a knowledge model able to predict what is called "light-limited growth" (Pruvost and Cornet, 2012; Takache et al, 2012) where biomass production rate is only a function of light received (no mineral limitations, optimal $\mathrm{pH}$, and temperature values). As discussed previously, appropriate engineering and operation of the cultivation system could make it possible to attain light-limited growth, but as culture systems can be limited by several other parameters, then quantitative information like biomass productivity will obviously be overpredicted. In some cases, this will be acceptable, as modeling is generally used to give a first estimation of process operation. In other cases, the model will have to be consolidated by adding equations related to effects of relevant parameters. However, as light will always influence growth (even in the case of severe limitation, like for nitrogen deprivation; see Kandilian et al, 2014), the model described in the following section will be able to serve as a basis for further model development work.

By definition, a light-limited growth model is able to couple light attenuation conditions to photosynthetic growth rate. This can prove invaluable when looking to further optimize the cultivation system, as it allows an indepth understanding of this coupling which governs the culture response. More practically, it also serves to determine information of primary relevance like time course of biomass concentration (or biomass productivity) as a function of microalgal cultivation systems design and operating parameters. The interested reader is invited to refer to Pruvost et al (2011a) for a fuller description of the solar PBR model and to further work by Pruvost et al (Pruvost and Cornet, 2012; Pruvost et al, 2011a, 2011b, 2012) for more detailed investigations. This model is the culmination of years of development and has proved efficient in several settings including artificial and sunlight conditions (Cornet and Dussap, 2009; Pruvost et al, 2011a, 2012, 2015; Takache et al, 2012) to the scaling and optimization of PBRs of various shapes (Cornet, 2010; Loubiere et al, 2011), biomass optimization of different microalga and cyanobacteria strains (Cornet and Albiol, 2000; Cornet et al, 1992b, 1998, 2003; Farges et al, 2009; Pruvost et al, 2011b; Takache et al, 2010).

\subsection{Overview of Light-Limited Growth Modeling in a PBR}

Takache et al (2012) introduced a generic model for light-limited growth in PBRs. This model was recently slightly revised to take into account the 
specific rate of photon absorption $A$ in place of irradiance $G$ which was found more relevant for coupling light absorption influence to photosynthetic growth response. Specific rate of photon absorption $A$ (Eq. 5) represents the light effectively absorbed by cells, which is the combination of light received (irradiance $G$ ) and ability of the cells to absorb light (absorption coefficient $\left.E a_{\lambda}\right)$.

The light-limited growth model is based on the coupling between a kinetic photosynthetic growth model and a radiative transfer model to represent light attenuation in a PBR volume as a function of parameters affecting light transfer, ie, biomass concentration, microalgae radiative properties, and light emission characteristics (spectrum, PFD, incident angle). The coupling between radiative and kinetic growth models makes it possible to calculate the resulting mean volumetric biomass production rate $\left\langle r_{X}\right\rangle$ and then biomass concentration and productivity. An overview of the model is given in Fig. 4. The following section gives details for each subpart of the model.

\subsection{Kinetic Growth Model}

In light-limited conditions, the kinetic growth model is able to relate the heterogeneous light radiation field in the PBR to local photosynthetic growth rate. Photosynthetic growth can be expressed first from the local specific rate of oxygen production or consumption $J_{\mathrm{O}_{2}}$, considered here at the scale of intracellular organelles, close to the primary photosynthetic and respiration events. When considering oxygen evolution/consumption, it is useful to introduce the compensation point of photosynthesis $A_{\mathrm{C}}$ (Cornet and Dussap, 2009; Cornet et al, 1992a; Takache et al, 2010). By definition, values of specific rate of photon absorption higher than $A_{\mathrm{C}}$ are necessary for a net positive photosynthetic growth (strictly, a net oxygen evolution rate). Values below the compensation point of photosynthesis have different effects depending on whether the cells are eukaryotic (microalgae) or prokaryotic (cyanobacteria). As cyanobacteria have their respiration inhibited by light, then for short-residence-time exposure to dark (Gonzalez de la Vara and Gomez-Lojero, 1986; Myers and Kratz, 1955), we can assume a nil oxygen evolution rate for irradiances below the $A_{\mathrm{C}}$ value. For eukaryotic microalgae, photosynthesis and respiration operate separately in chloroplasts and mitochondria. Hence microalgae, unlike cyanobacteria, present respiration both in the dark and in light. Oxygen consumption rates will thus be obtained for values below the compensation point of photosynthesis. 


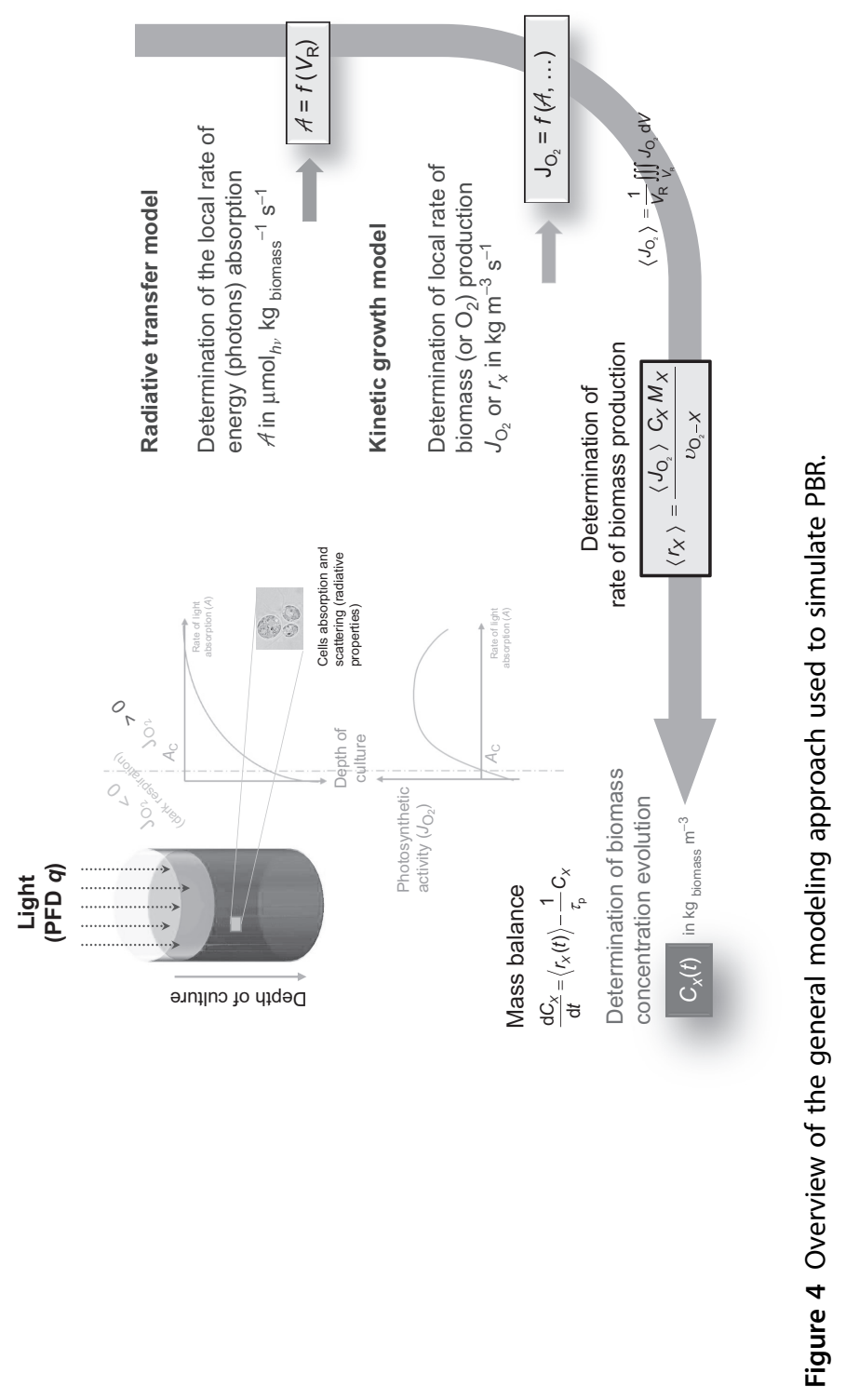


The kinetic response needs to be related to the heterogeneous light distribution in cultivation systems, represented here by the specific rate of photon absorption $A\left(\mu \mathrm{mol}_{h \nu} \mathrm{s}^{-1} \mathrm{~kg}^{-1}\right)$. As previously explained, Eq. (6) on the inhibition of respiration by light was proposed for cyanobacteria by Cornet and Dussap (2009):

$$
J_{\mathrm{O}_{2}}=\rho \bar{\varphi}_{\mathrm{O}_{2}}^{\prime} A \mathcal{H}\left(A-A_{\mathrm{C}}\right)=\rho_{\mathrm{M}} \frac{K}{K+G} \bar{\varphi}_{\mathrm{O}_{2}}^{\prime} A \mathcal{H}\left(A-A_{\mathrm{C}}\right)
$$

where $\mathcal{H}\left(A-A_{\mathrm{C}}\right)$ is the Heaviside function $\left(\mathcal{H}\left(A-A_{\mathrm{C}}\right)=0\right.$ if $A<A_{\mathrm{C}}$ and $\mathcal{H}\left(A-A_{\mathrm{C}}\right)=1$ if $\left.A>A_{\mathrm{C}}\right), \rho=\rho_{\mathrm{M}} \frac{K}{K+A}$ is the energetic yield for photon conversion of maximum value $\rho_{\mathrm{M}}$ (demonstrated as roughly equal to 0.8 ; Table 1), $\bar{\varphi}_{\mathrm{O}_{2}}^{\prime}=v_{\mathrm{O}_{2}-X} \bar{\varphi}_{X}^{\prime}$ is the molar quantum yield for the $Z$-scheme of photosynthesis as deduced from the structured stoichiometric equations (see Cornet et al, 1998; Pruvost and Cornet, 2012), and $K$ is the half-saturation constant for photosynthesis depending on the microorganism considered.

Takache et al (2012) completed this formulation for the specific case of microalgae with an additional term (right-hand term in Eq. 6) to consider respiration activity in light (Takache et al, 2012), which was to be found especially necessary if a dark zone appears in the culture volume due to the significant contribution of respiration to resulting growth in the whole PBR. By introducing the specific rate of photon absorption $\mathcal{A}$ in place of irradiance $G$, as explained earlier, Eq. (7) can thus be used for microalgae:

$$
\begin{aligned}
J_{\mathrm{O}_{2}} & =\left[\rho \bar{\varphi}^{\prime}{ }_{\mathrm{O}_{2}} \mathcal{A}-\frac{J_{\mathrm{NADH}_{2}}}{v_{\mathrm{NADH}_{2}-\mathrm{O}_{2}}} \times \frac{K_{\mathrm{r}}}{K_{\mathrm{r}}+\mathcal{A}}\right] \\
& =\left[\rho_{\mathrm{M}} \frac{K_{\mathrm{A}}}{K_{\mathrm{A}}+\mathcal{A}}{\overline{\varphi^{\prime}}}_{\mathrm{O}_{2}} \mathcal{A}-\frac{J_{\mathrm{NADH}_{2}}}{v_{\mathrm{NADH}_{2}-\mathrm{O}_{2}}} \times \frac{K_{\mathrm{r}}}{K_{\mathrm{r}}+\mathcal{A}}\right]
\end{aligned}
$$

where $J_{\mathrm{NADH}_{2}}$ is specific rate of cofactor regeneration on the respiratory chain, here linked to oxygen consumption by the stoichiometric coefficient $v_{\mathrm{NADH}_{2}-\mathrm{O}_{2}}$ (the stoichiometric coefficient of cofactor regeneration on the respiratory chain). Note that the effect, well known to physiologists, of the radiation field on the respiratory activity term was taken into account as an adaptive process of cell energetics (Cogne et al, 2011; Cournac et al, 2002; Peltier and Thibault, 1985). The decrease in respiration activity with respect to light was modeled here by an irradiance-dependent relation in a preliminary approach by simply introducing an inhibition term with a constant $K_{\mathrm{r}}$ describing the decreased respiration in light. We stress that this parameter is entirely determined by the knowledge of the compensation 
point of photosynthesis $A_{\mathrm{C}}\left(J_{\mathrm{O}_{2}}\left(A_{\mathrm{C}}\right)=0\right)$ when the specific respiration rate $J_{\mathrm{NADH}_{2}}$ is known (roughly equal to $14 \times 10^{-3} \mathrm{~mol}_{\mathrm{NADH}_{2}} \mathrm{~kg}_{X}^{-1} \mathrm{~s}^{-1}$, with $A_{\mathrm{C}}$ in the range $1500-3000 \mu \mathrm{mol}_{h \nu} \mathrm{kg}^{-1} \mathrm{~s}^{-1}$ for eukaryotic cells and 200$500 \mu \mathrm{mol}_{h \nu} \mathrm{kg}^{-1} \mathrm{~s}^{-1}$ for prokaryotic cells).

As a direct result of the light distribution inside the culture, the kinetic relation (Eq. 6 for cyanobacteria or Eq. 7 for microalgae) is of the local type. This implies calculating the corresponding mean value by averaging over the total culture volume $V_{\mathrm{R}}$ :

$$
\left\langle J_{\mathrm{O}_{2}}\right\rangle=\frac{1}{V_{\mathrm{R}}} \iiint_{V_{\mathrm{R}}} J_{\mathrm{O}_{2}} \mathrm{~d} V
$$

For a cultivation system with Cartesian one-dimensional light attenuation (such as flat-panel PBRs), this consists of a simple integration along the depth of culture $z$ :

$$
\left\langle J_{\mathrm{O}_{2}}\right\rangle=\frac{1}{L} \int_{z=0}^{z=L} J_{\mathrm{O}_{2}} \mathrm{~d} z,
$$

where $L$ is reactor depth. Once $\left\langle J_{\mathrm{O}_{2}}\right\rangle$ is known, the mean volumetric biomass growth rate $\left\langle r_{X}\right\rangle$ can be deduced directly as:

$$
\left\langle r_{X}\right\rangle=\frac{\left\langle J_{\mathrm{O}_{2}}\right\rangle C_{X} M_{X}}{v_{\mathrm{O}_{2}-X}}
$$

where $M_{X}$ is C-molar mass of the biomass and $v_{\mathrm{O}_{2}-X}$ is the stoichiometric coefficient of oxygen production (see Table 1 for an example of parameters set). Hence the mass balance equations (Eqs. 5 or 6 ) can be solved for any light-limited growth operating conditions.

Finally, once the mean volumetric growth rate is known, the resolution of the mass balance equation for biomass can serve to calculate biomass concentration and productivity as a function of operating parameter (lighting conditions and dilution rate $D$ - or residence time $\tau_{\mathrm{p}}=1 / D$-resulting from the liquid flow rate of the feed):

$$
\frac{\mathrm{d} C_{X}}{\mathrm{~d} t}=\left\langle r_{X}\right\rangle-\frac{C_{X}}{\tau_{\mathrm{p}}}
$$

\subsection{Modeling of Radiative Transfer}

Solving Eq. (6) or (7) entails determining the field of the specific rate of photon absorption $A$, which is obtained from radiative transfer modeling. This modeling is highly dependent on cultivation system geometry and can range 
from simple one-dimensional (Cornet, 2010; Pottier et al, 2005) to complex three-dimensional PBR geometries (Dauchet et al, 2013; Lee et al, 2014). Luckily, most cultivation systems present light attenuation along only one main direction (ie, the depth of culture $z$ ), which makes it possible to apply a hypothesis of one light attenuation direction and thus apply a simplified model like the two-flux model that has already proved efficient in several studies (Cornet et al, 1995, 1998; Lee et al, 2014; Takache et al, 2012).

A full description can be found in Pottier et al (2005) and Pruvost et al (2011a) for the more general case of solar irradiation (direct and diffuse radiation, non-normal incidence angle). A typical solution is given below as a function of the incident angle $\theta$ to take into account the general case of oblique irradiation with any incident light spectrum $(\cos \theta=1$ in the usual case of normal incidence):

$$
\frac{G_{\lambda}}{q_{\lambda}}=\frac{2}{\cos \theta} \frac{\left(1+\alpha_{\lambda}\right) \exp \left[-\delta_{\lambda}(z-L)\right]-\left(1-\alpha_{\lambda}\right) \exp \left[\delta_{\lambda}(z-L)\right]}{\left(1+\alpha_{\lambda}\right)^{2} \exp \left[\delta_{\lambda} L\right]-\left(1-\alpha_{\lambda}\right)^{2} \exp \left[-\delta_{\lambda} L\right]}
$$

where $q_{\lambda}$ is PFD measured perpendicular to the illuminated surface, $\alpha_{\lambda}=\sqrt{\frac{E a_{\lambda}}{\left(E a_{\lambda}+2 b_{\lambda} E s_{\lambda}\right)}}$ is the spectral linear scattering modulus (also see Table 1 for PAR-averaged values), and $\delta_{\lambda}=\frac{\alpha C_{X}}{\cos \theta}\left(E a_{\lambda}+2 b_{\lambda} E s_{\lambda}\right)$ is the spectral two-flux extinction coefficient, where $E a_{\lambda}$ and $E s_{\lambda}$ are spectral values of mass absorption and mass scattering coefficients, respectively, for the cultured photosynthetic microorganism, and $b_{\lambda}$ is the back-scattered fraction. As described elsewhere (Pottier et al, 2005; Soulies et al, submitted), Eq. (12) can be simplified for a spectrally averaged resolution to reduce the calculation effort.

Once the light attenuation profile is known, the illuminated fraction $\gamma$ can be obtained (Cornet et al, 1994; Takache et al, 2010). The illuminated fraction $\gamma$ is given by the depth of culture $z_{\mathrm{c}}$ where the value of specific rate of photon absorption for compensation $A\left(z_{\mathrm{c}}\right)=A_{\mathrm{C}}$ is obtained, with $A_{\mathrm{C}}$ being the minimum value required to obtain net positive photosynthetic growth ( $J_{\mathrm{O}_{2}}=0$ for $A=A_{\mathrm{C}}$ in Eq. 6). In the case of cultivation systems with one-dimensional light attenuation, we obtain:

$$
\gamma=\frac{V_{\text {light }}}{V_{\mathrm{r}}}=\frac{z_{\mathrm{c}}}{L}
$$

A $\gamma$ value below 1 indicates that all light available for a net photosynthetic growth is absorbed by the culture. Conversely, when the illuminated 
fraction is greater than 1 (a hypothetical representation because at maximum $V_{\text {light }}=V_{\mathrm{r}}$ ), some of the light is transmitted.

\subsection{Determination of Radiative Properties}

The radiative transfer calculation implies determining spectral radiative properties of microalgal suspension $\left(E a_{\lambda}, E s_{\lambda}, b_{\lambda}\right)$. Despite obvious relevance to PBR modeling (and engineering), these properties remain tricky to determine as there are still only a handful of studies proposing robust determinations. Determining these properties is effectively far from a trivial task. Future developments should solve this problem by providing engineers with approaches that are easier to apply in practice.

As things stand, there are two approaches. Radiative properties can be determined experimentally from measurements with aspectrophotometer equipped with an integrated sphere. This method was proposed by Pilon et al and has already been applied on several species (Berberoglu et al, 2008; Kandilian et al, 2013; Pilon et al, 2011). Alternatively, radiative properties can be determined from theory (see Dauchet et al, 2015 for a revised description). However, the input parameters are the pigment contents and the size distributions of the cells, which have to be determined experimentally. For example, it was shown that C. vulgaris and Chlamydomonas reinhardtii could be assimilated to spherical equivalent particles with an average radius of $2 \mu \mathrm{m}$. Size distribution was found to be log-normal with a standard deviation of $1.218 \mu \mathrm{m}$ for C. vulgaris and $1.17 \mu \mathrm{m}$ for C. reinhardtii (Pottier et al, 2005).

\subsection{Solar PBR Modeling}

Modeling is especially useful to solar culture as it can relate the complex phenomena involved in these conditions, such as time variations in sunlight in terms of intensity, beam-diffuse radiation partitioning, or collimated angle onto the PBR surface, and their effects on radiative transfer in the culture volume and the resulting photosynthetic conversion and biomass growth. Several recent studies have modeled solar PBR operation in attempts to optimize productivities as a function of PBR design, location, and/or cultivated species (Pruvost et al, 2011a, 2012; Quinn et al, 2011; Slegers et al, 2011, 2013a, 2013b). In general, current models mainly aim to relate sunlight conditions obtained from meteorological databases to growth kinetics in order to predict PBR performances (Pruvost et al, 2011a; Quinn et al, 2011; Slegers et al, 2011). These models can provide valuable predictions of productivity for PBRs operated during an entire year. They 
can also assess the influence of various parameters, such as PBR location, harvesting strategy, strains cultivated, and the effects of night and day cycles. However, they may be regarded as oversimplified given the complexity and numbers of different parameters affecting PBR operation and productivity in outdoor conditions. As discussed earlier, numerous features can impair bioprocess production, from mineral or carbon limitation to nonideal temperature or $\mathrm{pH}$ control, nonoptimized harvesting strategies, contamination, and more. There is a clear need to pursue with efforts to develop a set of robust tools for solar cultivation optimization to achieve better accuracy and extend their applicability to other solar PBR-related challenges. For example, Slegers et al (2013a) integrated a thermal model able to predict the time-course evolution of culture temperature under solar conditions and assess its influence on growth. Temperature was found to strongly influence growth rate and the resulting biomass productivity.

For the so-called light-limited regime where only light limits growth, the model can be adapted to solar case. The main modifications compared to artificial light reside in the consideration of sunlight characteristics (nonnormal incidence, direct and diffuse light) in radiative transfer calculation and the need to introduce a time resolution due to the time-changing irradiation conditions, with day and night periods requiring special consideration. This model is already described elsewhere (Pruvost and Cornet, 2012; Pruvost et al, 2011a, 2012, 2015), so only the main features are reported here. Note that all these features were proved relevant in the predictions of solar PBR performances.

The example in this section applies to cultivation systems presenting a flat illuminated surface (ponds, rectangular PBR, etc.). The one-dimensional and azimuth-independence assumptions can then be used to describe the irradiance field in the culture bulk, making it possible to apply the two-flux radiative model with its corresponding analytical solutions (Pottier et al, 2005). Application to the solar case implies factoring in non-normal incidence (thus introducing the incident angle $\theta$ ) with a separate treatment of the direct and diffuse components of the radiation due to their difference in angular distribution over the PBR surface (Pruvost et al, 2011a). Total hemispherical incident light flux density (or PFD, see next section) $q$ is divided into direct $q_{/ /}$and diffuse $q_{\cap}$ components $\left(q=q_{/ /}+q_{\cap}\right)$. Total irradiance (representing the amount of light received in the culture bulk) is given by summing the resulting contribution of collimated and diffuse radiation:

$$
G(z)=G_{\mathrm{col}}(z)+G_{\mathrm{dif}}(z)
$$


where $G_{\mathrm{col}}$ is the irradiance field for collimated radiation, as given by:

$$
\frac{G_{\mathrm{col}}(z)}{q_{/ /}}=\frac{2}{\cos \theta} \frac{(1+\alpha) \exp \left[-\delta_{\mathrm{col}}(z-L)\right]-(1-\alpha) \exp \left[\delta_{\mathrm{col}}(z-L)\right]}{(1+\alpha)^{2} \exp \left[\delta_{\mathrm{col}} L\right]-(1-\alpha)^{2} \exp \left[-\delta_{\mathrm{col}} L\right]}
$$

and $G_{\text {dif }}$ the irradiance field for diffuse radiation:

$$
\frac{G_{\text {dif }}(z)}{q_{\cap}}=4 \frac{(1+\alpha) \exp \left[-\delta_{\text {dif }}(z-L)\right]-(1-\alpha) \exp \left[\delta_{\text {dif }}(z-L)\right]}{(1+\alpha)^{2} \exp \left[\delta_{\text {dif }} L\right]-(1-\alpha)^{2} \exp \left[-\delta_{\text {dif }} L\right]}
$$

In these equations, $\delta_{\mathrm{col}}=\frac{\alpha C_{X}}{\cos \theta}(E a+2 b E s)$ and $\delta_{\text {dif }}=2 \alpha C_{x}(E a+2 b E s)$ are the two-flux collimated and diffuse extinction coefficients, respectively.

Determining the irradiance field makes it possible to determine the corresponding local photosynthetic growth rate in the culture volume. The same kinetic relations (Eq. 6 or 7) can be applied here, making it possible to calculate mass volumetric biomass growth rate $\left\langle r_{X}\right\rangle$ (Eq. 11). The only restriction is that Eqs. (6) and (7) are valid insofar as the culture is illuminated (ie, during daytime). At night, long dark periods of several hours trigger a switch to respiratory metabolism which results in biomass catabolism (Le Borgne and Pruvost, 2013; Ogbonna and Tanaka, 1996). This biomass catabolism is species dependent and differs strongly between eukaryotic (microalgae) and prokaryotic (cyanobacteria) cells. For Arthrospira platensis and $C$. reinhardtii, values of $\left\langle r_{X}\right\rangle / C_{X}=\mu=0.001$ and $0.004 \mathrm{~h}^{-1}$, respectively, were recorded at their optimal growth temperature, ie, $308 \mathrm{~K}$ for $A$. platensis and 293K for C. reinhardtii (Cornet, 1992; Le Borgne, 2011).

Finally, the determination of the mean growth rate allows the mass balance equation, here for biomass, to be solved (Eq. 11). The variable PFD in sunlight conditions means that the irradiance field inside the culture bulk and the resulting local and mean volumetric growth rates vary continuously, and hence steady state cannot be assumed in Eq. (11). This implies solving the transient form of the mass balance equation. Once the time course of biomass concentration has been determined, the corresponding biomass productivity can be calculated, as well as surface productivity $P_{\mathrm{S}}\left(\mathrm{g} \mathrm{m}^{-2}\right.$ day $\left.{ }^{-1}\right)$ which is a useful variable to extrapolate to land-area production (Eq. 2).

\section{OPTIMIZATION OF PBR OPERATION}

\subsection{Understanding Light-Limited Growth}

In practice, the control of culture conditions such as $\mathrm{pH}$ and temperature can prove challenging, especially in outdoor conditions (Borowitzka, 1999; 
Grobbelaar, 2008; Richmond, 2004a; Torzillo et al, 1996). These challenges can, however, be overcome with adequate engineering and control of the cultivation system. As technical solutions are highly dependent on culture system technology, these aspects will not be discussed in detail here. The main point is that if all cultivation conditions are kept at optimal values and nutrients are provided in adequate amounts, then light-limited conditions should eventually occur, which is crucial given that the light-limited regime has several major features.

The first consequence of light-limited conditions achievement is that, by definition, the culture is not subject to any further limitation other than light use. Thus, maximum biomass productivity can be achieved and is determined by the amount of light provided and its use by the culture (Pruvost, 2011; Pruvost and Cornet, 2012; Pruvost et al, 2011b, 2012; Takache et al, 2010). Any limitation other than light limitation would result in further decreases of biomass productivity, whereas maximizing the PFD received on the culture system increases its productivity. Note that this remains valid in the case of high PFD leading to photoinhibition of the photosynthetic apparatus (PFD grossly greater than $400 \mu \mathrm{mol}_{h \nu} \mathrm{m}^{-2} \mathrm{~s}^{-1}$ ). Special attention should be paid to light attenuation conditions to avoid or at least greatly reduce photoinhibition phenomena by operating the PBR to achieve complete light extinction in the culture, as described in detail in the next section.

A second important consequence is that in the light-limited regime, controlling the incident light and its effect on the process equates to controlling aggregate cultivation system performance. This is the so-called physical limitation in chemical engineering, where the process is limited by one parameter which, if controlled, enables control of the entire process. This feature is essential to the efficient design and operation of photobiological cultivation systems. The role of light in the rational design of microalgal cultivation systems has been touched on earlier and will be explored in greater depth later in this chapter by actual examples of technologies. Implications in terms of operation are discussed later.

\subsection{Optimizing Light Attenuation Conditions for Maximal Biomass Productivities in PBRs}

Although a necessary condition, the light-limited regime alone is not sufficient to obtain maximal biomass productivities, which also hinge on controlling radiative transfer conditions inside the culture (Cornet and Dussap, 2009; Pruvost, 2011; Takache et al, 2010). As already discussed, 
if biomass concentration is too low, some of the light gets transmitted through the culture, and if biomass concentration is too high, a dark zone appears deep in the culture. For eukaryotic cells like microalgae that demonstrate respiration in light, a dark zone in the culture volume where respiration is predominant will result in a loss of productivity due to respiratory activity. Maximal productivity will then require the specific condition of full absorption of all light received but without a dark zone in the culture volume-in other words the luminostat regime (Pruvost and Cornet, 2012; Takache et al, 2010). As a result, unlike processes based solely on surface conversion (eg, photovoltaic panels), optimizing the amount of light collected on the microalgal cultivation system surface is still not sufficient. As light conversion by photosynthetic microorganisms occurs within the culture bulk, transfer of the collected light flux inside the bulk has to be taken into account.

Light attenuation conditions can be controlled by adjusting biomass concentration in the cultivation system (see Section 2.1.2.5), which can be done in continuous mode by modifying the residence time $\tau_{\mathrm{p}}$ applied to the system (or dilution rate $D=1 / \tau_{\mathrm{p}}$ ). In practice, maintaining optimal light attenuation conditions is no easy task, especially in the case of solar production which adds a degree of complexity to the optimization and control of the cultivation system compared to artificial illumination. The process is fully dynamic and driven by an uncontrolled input, ie, solar incident flux. Under sunlight, biomass growth rate is insufficient to compensate for the rapid changes in sunlight intensity. Consequently, light attenuation conditions that are fixed by biomass concentration are never optimal. A compromise has to be found on the conditions thus applied, for example, by defining a residence value that will maximize biomass productivity over the period of operation by acting on biomass concentration time course and the related light attenuation conditions.

\subsubsection{The Role of Light Attenuation Conditions in Culture Stability}

Although a dark volume has an impact on respiration activity (see next), high light attenuation conditions are also well known to have a positive effect on culture stability (Carvalho et al, 2011; Grima et al, 1999; Hindersin et al, 2013; Richmond, 2004b; Torzillo et al, 1996). Light transmission also corresponds to a high light received per cell (ie, high specific absorption rates, see Section 2.1.2.5), which could induce culture drift by oversaturation of the photosynthetic chain (Grima et al, 1996; Hindersin et al, 2013; $\mathrm{Wu}$ and Merchuk, 2001). Note also that this generally also results in 
photoacclimation and a decrease of pigment content (Zonneveld, 1998), which in turn increases light penetration in the culture depth, and thus the light received per cell, thereby increasing culture drift. In practice, the culture will become highly unstable when transmission occurs, especially if PFD is higher than $200 \mu \mathrm{mol}_{h \nu} \mathrm{m}^{-2} \mathrm{~s}^{-1}$. A typical result is given in Fig. 5, which depicts a $C$. vulgaris culture in a lab-scale PBR. The same PFD is applied in all experiments, and only light attenuation conditions are modified through changing biomass concentration by adjusting residence time. In the light transmission conditions, pigment content decreased as a result of a higher specific photon energy absorption rate. This decrease was especially visible for chlorophylls, where it results in a higher carotenoids-tochlorophylls ratio (as shown by the yellow (light gray in the print version) color of the culture). In practice, it also generally marks the appearance of biofilm, despite the lower biomass concentration obtained, leading to a progressive culture drift up to potential washout.

For the operator, a general rule will be to promote full-light attenuation conditions. Note that this condition will be difficult to fulfill in some cases, such as in species presenting low pigment content (such as strains with small

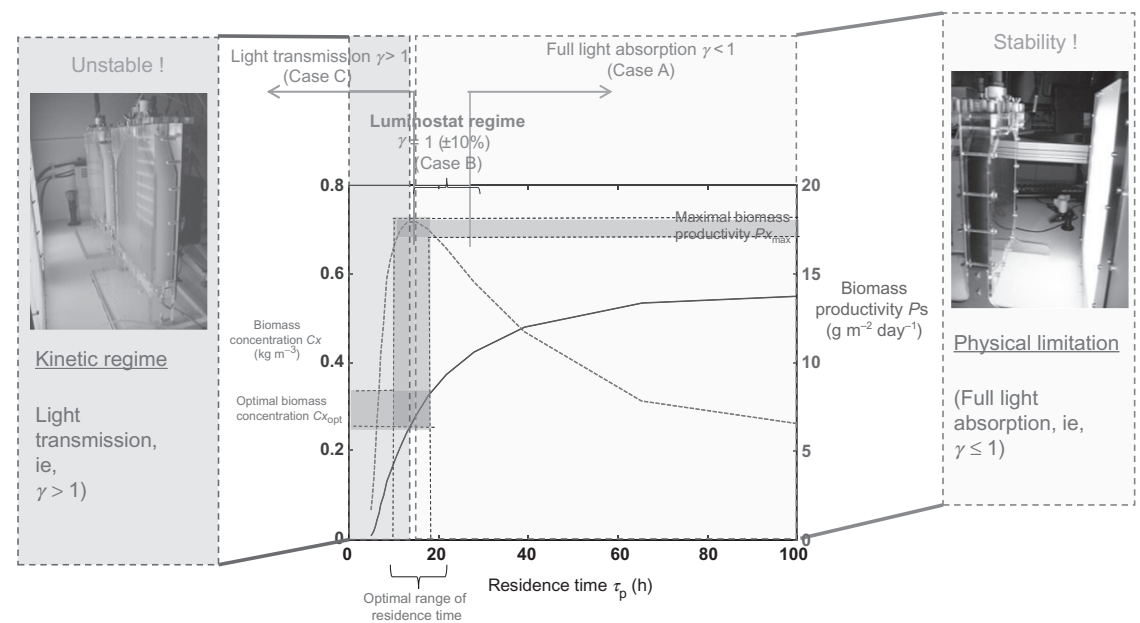

Figure 5 Effects of light attenuation conditions on culture stability and biomass productivity. For low residence time, low biomass concentration results in light transmission and high rate of photon absorption (ie, high light received per cell) inducing possible culture drift. For high residence time, the dark volume then generated can have a negative effect on biomass productivity due to the promotion of respiration activity, but also results in more stable culture because of a lower rates of photon absorption (ie, lower light received per cell). 
antennas; Berberoglu et al, 2008) due to their lower absorption, or in solar conditions due to the time-course changes in light attenuation conditions, as we will see later.

\subsubsection{Microalgae vs Cyanobacteria}

If the biomass is too high, a dark zone appears in the culture. Here is an important distinction to make between eukaryotic (microalgae) and prokaryotic (cyanobacteria) cells. In cyanobacteria cultivation, as the cells have common electron carrier chains and no short-time respiration in the dark (Gonzalez de la Vara and Gomez-Lojero, 1986), a dark zone will be sufficient $(\gamma \leq 1)$ to guarantee maximal productivity (Cornet, 2010; Cornet and Dussap, 2009). For eukaryotic cells presenting respiration in light (microalgae), a dark zone in the culture volume where respiration is predominant will result in a loss of productivity due to biomass catabolism. Achieving maximal productivity will thus be contingent on the $\gamma$ fraction meeting the exact condition $\gamma=1$ (the "luminostat" regime), corresponding to full absorption of all light received but without a dark zone in the culture volume (Takache et al, 2010).

In practice, maintaining an optimal value of the $\gamma$ parameter is not easy, especially with microalgae (where the condition $\gamma=1$ has to be met). Some illustrations are given below for both batch and continuous production modes. Because the regime does not allow full absorption of the light captured, light transmission always leads to a loss of efficiency, in addition to possible culture drift due to an excess of light received per cell, as discussed earlier $(\gamma>1)$. This regime is, however, usually encountered at the beginning of a batch production run (Fig. 6A). Biomass growth means that the light attenuation conditions will continuously evolve and the $\gamma$ value will progressively decrease down to a value below 1. For prokaryotic cells, as soon as full absorption is obtained, the maximal value of the mean volumetric growth rate will be achieved and then remain constant (until a large dark zone is formed, inducing another possible shift in cell metabolism). For eukaryotic cells, the condition $\gamma=1$, and thus the maximal value of the mean volumetric growth rate $\left\langle r_{X}\right\rangle$ will only be transitorily satisfied (mean volumetric growth rate being represented by the slope of $C_{X}(t)$, see Eq. 11 with $\left.1 / \tau_{\mathrm{p}}=0\right)$. The increase in the dark volume will then progressively decrease the mean volumetric growth rate.

In continuous mode, light attenuation conditions can be controlled by modifying the dilution rate to adjust the in-system biomass concentration. For cyanobacteria (Fig. 6B), there will be an optimal range of biomass 

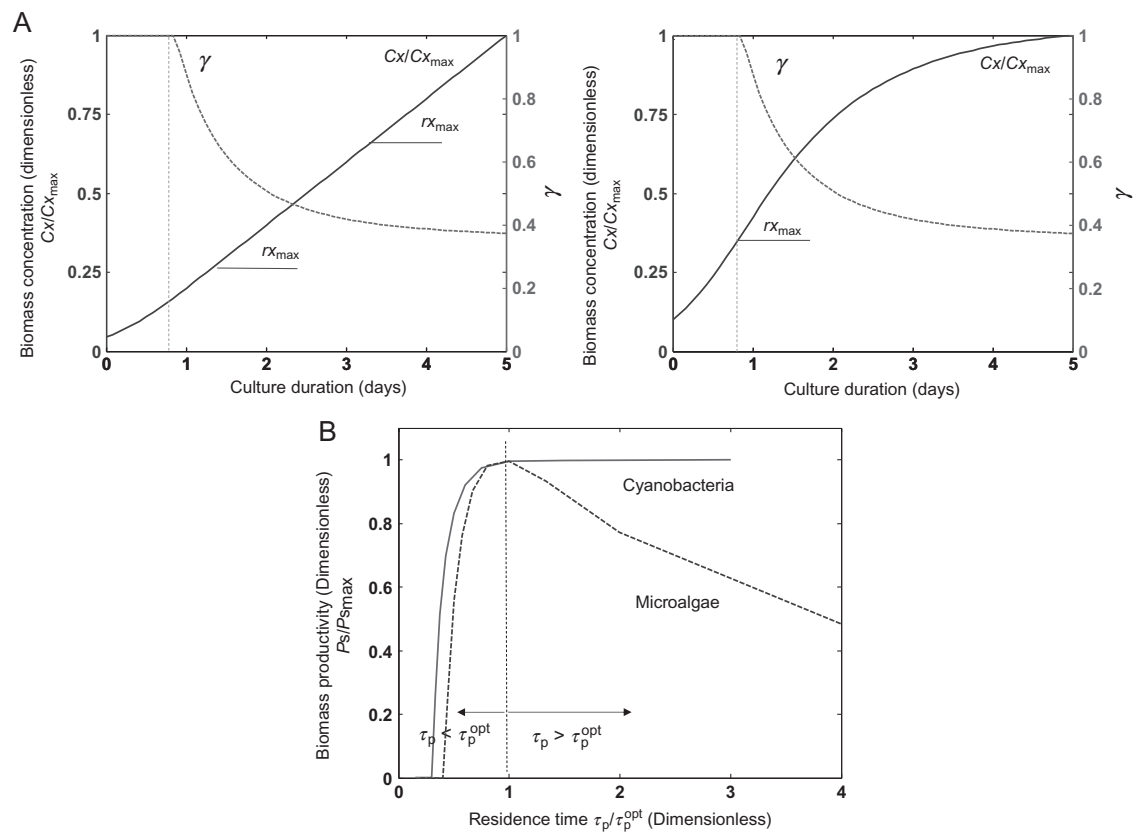

Figure 6 Typical evolution of biomass concentration during a batch cultivation of cyanobacteria and microalgae in light-limited conditions (A). Biomass productivity and concentration in continuous mode are given in (B).

concentrations to meet the condition $\gamma \leq 1$. For microalgae, the $\gamma=1$ condition will require an optimal biomass concentration $\left(C_{X}^{\text {opt }}\right)$ corresponding precisely to the condition of full-light absorption but no dark zone (as shown in Takache et al, 2010, a deviation of the $\gamma$ value in the range $\gamma=1 \pm 15 \%$ is tolerable in practice).

Whichever production mode (continuous or batch) is used, the control of light attenuation conditions, represented here by the illuminated fraction (with $\gamma \leq 1$ for cyanobacteria and $\gamma=1 \pm 15 \%$ for microalgae), makes it possible to obtain the maximum biomass productivity of the cultivation system in light-limited conditions (volume and surface). If radiative transfer conditions are known (using a radiative transfer model, as already described), then the optimal biomass concentration can be determined theoretically, or else experimentally simply by varying the residence time and measuring corresponding biomass concentration and productivity (Takache et al, 2010).

\subsection{Optimizing Light Attenuation in Solar Cultivation}

Outdoor conditions and the use of sunlight as primary energy source pose several challenges to the engineering design and control of outdoor 
cultivation systems. Sunlight is characterized by a wide, rapid, and uncontrolled variation in irradiation conditions. On a single day, the PFDs received onto a cultivation system surface can range from null (night) to potentially damaging levels for the photosynthetic chain of growing cells (high PFDs typically larger than $1000 \mu \mathrm{mol} \mathrm{m}^{-2} \mathrm{~s}^{-1}$, which are commonly encountered in most locations on Earth in summer). Strong light attenuation in the PBR is in this case known to have a positive effect as it decreases the amount of light energy received per cell along the depth of the PBR (Carvalho et al, 2011; Hindersin et al, 2013; Torzillo et al, 1996).

The amount of direct and diffuse solar incident irradiance as well as the strongly time-dependent incident PFD and the associated incident angle have also been found to significantly dictate process efficiencies (Pruvost et al, 2011a, 2012). Consequently, although the luminostat regime is the ideal case leading to maximum biomass productivity, it cannot be maintained under solar conditions due to how much faster light varies with time than biomass concentration (Hindersin et al, 2013; Pruvost et al, 2011a, 2012). The net result is that there is a design and operation compromise to be found.

In continuous or semi-continuous PBRs, this can be achieved by defining, for example, a residence time that maximizes yearly biomass productivity through control over the temporal evolution of the biomass concentration and light attenuation in the PBR. Modeling can prove invaluable here by simulating PBR operation over a whole-year period as a function of various key parameters such as (1) PBR location, design, inclination, and orientation; (2) PBR operating parameters (harvesting strategy for instance); and (3) species cultivated.

Fig. 7 gives examples of yearly biomass productivities as a function of residence time applied on the cultivation system (see Pruvost et al, 2015 for details). As was the case for continuous light, an optimal value exists, but it corresponds to the value that gives the maximal productivity over a given time period. Simply optimizing the residence time in the cultivation system is not enough to maintain the ideal luminostat regime condition $(\gamma=1)$ because the illumination conditions vary so much faster than the kinetics of photosynthetic growth. The optimal residence time can only be regarded as the best compromise to maximize productivity on a given cultivation period (a full year period here). The immediate consequence is that it will result in large variation of light attenuation conditions with time in the cultivation system.

Obviously, the residence time value can be optimized all along the year. In winter, for example, increasing the residence time can prove beneficial for 


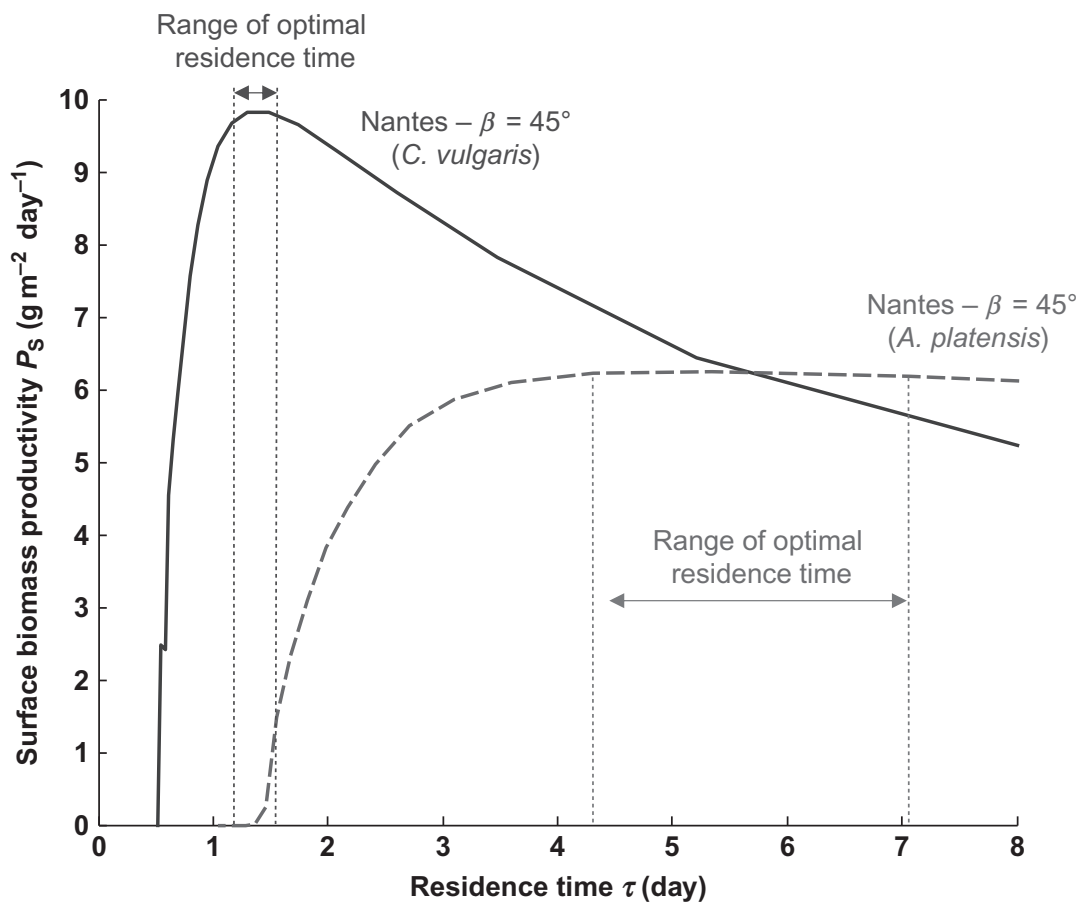

Figure 7 Yearly average areal productivity of an inclined flat-panel PBR (45 degree) as a function of the residence time applied on the cultivation system operated in continuous mode (Nantes locations, France). Values are given for the microalga C. vulgaris and for the cyanobacteria A. platensis, illustrating the narrower range of residence time to maximize productivity for eukaryotic cells as explained by their sensitivity to dark volumes induced by too high values of residence time values.

microalgae due to their lower growth, which means longer residence times for this specific period can have positive impacts on net biomass productivity. Modeling is again valuable here, as it can be used to calculate biomass productivity for any residence time value and to define an optimal year-long residence time course. Looking at the C. vulgaris growth presented in Fig. 7, ideally, higher values should have been applied in winter (up to $\tau_{\mathrm{p}}=2.3$ days), and lower values applied in summer (down to $\tau_{\mathrm{p}}=0.8$ day).

Fig. 7 also compares biomass productivities between microalgae (ie, C. vulgaris) and cyanobacteria (ie, A. platensis). The same type of evolution is achieved for both species at low residence times (rapid decrease of surface productivity toward culture washout for low residence time values, ie, high dilution rate), but C. vulgaris showed significantly different productivity at high residence time values whereas $A$. platensis showed little impact. 
As a consequence, maximum values of surface productivities for C. vulgaris were only found for a narrow range of residence times. This important difference between the two microorganisms is explained by the negative influence of dark volume on microalgae growth kinetics. High residence times result in higher biomass concentrations and light attenuation conditions. As already observed in continuous light conditions, the impact is negligible for cyanobacteria but not for microalgae due to their respiration activity in the dark. This result has important practical implications: a harvesting strategy that maximizes biomass productivity is fairly easy to find for cyanobacteria $\left(\tau_{\mathrm{p}} \leq \tau_{\mathrm{p}}^{\text {opt }}\right)$ but very difficult to find for the microalga $C$. vulgaris.

Another important issue resides in the light regimes obtained in the culture volume when operated in solar conditions. Once the residence time is defined, the year-long time course of biomass concentration can be calculated and thus the corresponding time evolutions of light attenuation conditions. Variations in incident irradiation mean that a wide range of light attenuation conditions can be encountered inside the culture volume over the course of a day, which can affect process stability, as described in Pruvost et al (2015, in press). As shown in Section 2.1.2.5, harvesting strategy (ie, residence time) will directly affect these light regimes. For example, promoting a higher residence time will increase biomass concentration and light attenuation (ie, decreasing photon absorption rates). This could reveal beneficial for periods where oversaturating light is encountered, such as at noon in summer. However, as increasing light attenuation conditions could also result in a decrease in biomass productivity, particularly with species that show significant respiration activity under illumination, then it will almost certainly be necessary to find a trade-off between process productivity, stability, and robustness. Here again, models can help. Modeling combined with in-depth investigations of the effect of oversaturating light on culture stability could serve as a foundation to advanced control strategies able to maintain the appropriate trade-off between biomass productivity maximization and robust culture operation, which is currently a big challenge for optimal solar culture system operation.

\section{DEVELOPMENT OF COMMERCIAL TECHNOLOGIES
BASED ON PBR ENGINEERING RULES}

\subsection{Introduction}

There is a wide variety of PBR technologies available, including tubular, cylindrical, and flat-panel systems (some examples are given in Fig. 2). This 
diversity of PBR designs is the result of various attempts to optimize light capture while satisfying other practical constraints related to (1) engineering design, including system integration, scale of production, materials selection, and project costs; and (2) system operation factors such as $\mathrm{CO}_{2}$ bubbling, oxygen removals, temperature and $\mathrm{pH}$ regulation, nutrient delivery. The literature counts an array of reports and publications on the various PBR technologies available (Borowitzka, 1999; Carvalho et al, 2006; Grima et al, 1999; Morweiser et al, 2010; Pruvost, 2011; Pulz, 2001; Ugwu et al, 2008), all of which have advantages and limitations in terms of control of culture conditions, culture confinement, hydrodynamic conditions, scalability, construction cost, biomass productivity, and energy efficiency. Regardless of the PBR concept employed, the goal is to provide sufficient control of the culture conditions to make the process only limited by the amount of light supplied and the photosynthetic process in the culture (ie, the "light-limited" regime presented in Section 3.1). PFD incident onto the PBR surface and PFD locally available inside the culture are major parameters. Although maximizing light intercepted is an obvious consideration of any microalgal cultivation system (as with any light-driven process), there are other constraints to also consider. For example, using the airlift method for mixing will preclude horizontal geometries. Shading needs to be accounted for when arranging vertical or tilted solar systems on a given land area. As a result, what characterizes microalgae culture more than any other bioprocess is the wide range of constraints involved, from light use optimization to cost of production, which ultimately makes an optimal culture technology impossible to define. These features have fueled the idea that the development of microalgal culture systems is more or less empirical. Nevertheless, as seen earlier in this chapter, there are several engineering tools now available, making it possible to propose rational and robust methods for the design of optimal geometries taking into account the application constraints. This is illustrated in the following sections by specific examples of PBR developments. The examples come from a community of groups that now share the same engineering tools (ie, as tools described here). Some of these examples are still promising lab-scale prototypes, while others are industrial units commercialized by French company AlgoSource Technology (www.algosource.com).

\subsection{Artificial Light Culture Systems}

\subsubsection{Lab-Scale Technologies}

Lab-scale technologies are very useful for fundamental studies as well as in investigations for parameter-setting process models or operating protocols 
for cultivation system scaling and optimization. The main constraint in the lab-scale design is to achieve well-controlled conditions, with the appropriate scale of production to allow sufficient sampling during the experiment (usually leading to around $1 \mathrm{~L}$ of culture volume). Note that the setting of experiments in well-controlled conditions is a general constraint of any kind of biological study. This is usually achievable in simpler systems, such as flasks (ie, studies on bacteria and yeasts for example), but in studies on photosynthetic microorganisms, the strong influence of light supply and especially light attenuation conditions makes it difficult to use flask-type systems for well-defined lab-scale investigations. At best, PFD received on the flask surface can be defined as an operating parameter. However, as seen many times above, growth rate is not only a function of PFD but also of light attenuation conditions, which are nigh impossible to determine in flask systems (3D geometries). Ideally, for investigations on photosynthetic microorganisms, engineers should opt for geometries enabling to control (and ideally, determine) light attenuation in the culture volume. Lab-scale systems should then be required to fulfill this condition for accurate and representative results. Note that this consideration applies not only to bioprocess investigations (ie, PBR optimization) but also to fundamental biology investigations where photosynthesis and thus light absorbed is a relevant factor (ie, most studies devoted to photosynthetic microorganisms).

\subsubsection{The Torus-Shaped PBR}

The torus-shaped PBR is a typical example of a microalgal culture system designed for lab-scale experiments requiring firm control of culture conditions (Fig. 2). The main characteristic is its torus shape, as the culture is circulated by the rotation of a marine impeller. The combination of the impeller and the loop configuration of the torus geometry allows good mixing without dead volume while keeping shear stress in a reasonable range (Pruvost et al, 2006). The light-supplying device (LED panel) is placed in front of the PBR. The plane front surface and the square-sectioned torus channel mean that the PBR presents no curved surfaces perpendicular to the light source. This prevents optical distortion along the light emission direction, which enables easy calculation of light transfer, as light attenuation occurs along only one main direction, and leads to the so-called onedimensional hypothesis. This configuration makes it possible to accurately determine light attenuation conditions (using Eq. 12 for example) and then local rate of photon absorption (Eq. 5), which can be related to photosynthetic growth (Eq. 7) or averaged over the culture volume to investigate its role as a process parameter (Kandilian et al, 2014). 
The torus-shaped PBR has been used for several studies in recent years, both to model and optimize microalgal biomass productivity (Takache et al, 2010, 2012) and to investigate the coupling between hydrodynamics and photosynthetic conversion (the "light/dark cycles effect"; Takache et al, 2015). As it employs mechanical mixing, the torus-shaped PBR was also found valuable for studies requiring accurate gas analysis. When combined with online gas analysis, the obtained setup was able to yield kinetics information on culture evolution as a function of culture conditions, which was impossible or at least less accurate with air injection-mixed technologies (ie, airlift PBR) due to gas dilution with the gas-flow carrier. A typical example is the investigations on $\mathrm{H}_{2}$ production from $C$. reinhardtii (Fouchard et al, 2008), where online monitoring of gas released and consumed $\left(\mathrm{O}_{2}, \mathrm{CO}_{2}\right.$, $\mathrm{H}_{2}$ ) combined with biotic-phase (total biomass and biomass composition in sugars, proteins, lipids, pigments) and abiotic-phase (carbon and mineral compound consumption) measurements made it possible to determine the effects of sulfur deprivation to induce anoxic conditions and starch accumulation and subsequently $\mathrm{H}_{2}$ production by microalgae. This work enabled a kinetic model to be set that, when combined with the highly controllable conditions of the torus-shaped PBR, led to the development of an optimized $\mathrm{H}_{2}$ production protocol (Degrenne et al, 2008, 2010, 2011a, 2011b; Fouchard et al, 2005, 2009). This setup was recently extended to the investigation of $\mathrm{CO}_{2}$ fixation by microalgae (Le Gouic, 2013). Another example of the use of the torus-shaped PBR can be found in Martzolff et al (2012). The good mixing performance, and especially the plug-flow behavior encountered in the torus loop, was used for isotopic nonstationary 13Cmetabolic flux analysis. This enabled the characterization of the kinetics of 13C-labeling incorporation, which helped to define the biochemical reaction network of C. reinhardtii (Cogne et al, 2011).

All those examples illustrate the large interest of using lab-scale PBR presenting a high control of culture conditions for fundamental studies, which encircles in-depth investigations of microalgae metabolism and physiology, and the setting and optimization of culture protocol for applications of interest.

\subsubsection{Efficient Overproducing Screening System-Photobioreactor}

A cultivation system specially adapted to screening microalgae in reliable conditions was recently developed (Fig. 2). This system, named efficient overproducing screening system-photobioreactor (EOSS-PBR), was used to evaluate cell growth and productivity to compare strain performances 
or characterize the effects of specific culture conditions on a given strain culture. It consists of six small-scale PBRs (bubble columns) operated in parallel. Each tube has a volume $V_{\mathrm{r}}=30 \mathrm{~mL}$ and an illuminated area $S_{\mathrm{L}}=0.008 \mathrm{~m}^{2}$. The EOSS-PBR was fully automated in terms of medium injection and biomass harvesting to allow semi-continuous cultivation with the ability to set different feeding or harvesting sequences in each tube.

EOSS-PBR enables both batch and semi-continuous cultivation. Semicontinuous cultivation was found relevant for allowing strains to progressively adapt to the growth conditions of PBR cultivation. For example, after receiving strains from collections, it was necessary to wait for several weeks before reaching a stable biomass production, indicating progressive adaptation of the cultivated strain to the conditions applied. The same behavior was observed when comparing various growth media on a given strain. As a result, this simple and easy-to-use system was found useful for adapting strains to PBR growth conditions to compare algae performances in reliable conditions. Examples of its use can be found in Taleb et al (2015).

\subsection{Industrial Technologies}

\subsubsection{Introduction}

The state of art in industrial-scale technologies (Carvalho et al, 2006; Janssen et al, 2003) covers a broad gamut of geometrical configurations, led by pneumatically agitated vertical column reactors, tubular reactors, and flat-panel reactors. The main reason for this diversity is that there is still no ideal starting geometry for microalgal culture systems, as illustrated here with two examples of artificial light PBRs designed by researchers and marketed by AlgoSource for different practical applications.

\subsubsection{Multimodule External-Loop Airlift PBR for Hatcheries}

The first culture system was designed for continuous microalgal production in mollusk hatcheries. The context of mollusk hatcheries poses two main constraints on PBR design. First, the microalgae commonly cultivated for mollusks are usually stress-sensitive species, such as Isochrysis affinis galbana, Chaetoceros calcitrans, Thalassiosira pseudonana, Skeletonema marinoi (Borowitzka, 1997). Second, microalgal production in hatcheries is mainly performed by batch cultures in systems consisting of vertical aerated column reactors. Their major drawbacks are only partial control of biomass quality and quantity, low productivity, contamination, manpower intensiveness (frequent handling and cleaning operations), and biofouling. To improve this situation, the PBR project brief was to enable continuous production 
adjustable to hatchery requirements, in a closed and artificially lit system, with the simplest possible design to minimize manufacturing cost, price, and floorspace; to be robust (marine atmosphere); and easy to clean. To respond to these constraints, the PBR consisted of a succession of elementary modules, each composed of two transparent vertical interconnected columns. Liquid-phase circulation was performed pneumatically, ie, by gas injectors placed at the column bottom and uniformly dispatched across the whole PBR. Each elementary module can thus be seen as an externalloop airlift PBR, except that the outlet of the downcomer is connected to the adjacent module. In addition, as already used in annular PBRs (MullerFeuga et al, 2003a, 2003b; Pruvost et al, 2002a) and torus PBRs (Pottier et al, 2005; Takache et al, 2010), a swirling motion was generated (tangential inlets) in order to minimize biofilm formation on the walls while keeping shear stress within a reasonable range. As a result, a PBR technology adapted to the specific case of microalgal production in mollusk hatcheries was proposed. The major utility of this design was not only to enable easy continuous microalgal culture in a closed, artificially illuminated system but also to offer volume modularity without scale-up/down calculations. Indeed, the illumination device was designed so as to conserve the same illuminated specific surface (ratio of illuminated surface to culture volume) whatever the number of modules, implying that, for a given incident flux and a fixed dilution rate, volumetric productivity remains identical for either a monomodule or multimodule PBR. Consequently, the number of elementary modules is only dictated by the microalgal production required. A complete description of the design procedure can be found in Loubiere et al (2009).

\subsubsection{Two-Side Illuminated Flat-Panel Airlift PBR}

The culture system described earlier is based on cylindrical tubes, which makes it difficult to calculate radiative transfer in the culture volume, which has to be solved numerically (Lee et al, 2014). As already described, the "one-dimensional hypothesis" where light attenuation occurs along only one main direction serves to obtain analytical relations to represent the light attenuation field (as with the two-flux model, Eq. 12). This enables accurate and easy determination of light attenuation conditions for any operating conditions and thus greater system control. Based on this statement, researchers designed a specific PBR. Like the multimodule external-loop airlift PBR, this system is of industrial size $(130 \mathrm{~L})$, but the unit is a flat panel with front illumination so as to respond to the one-dimensional hypothesis. It is also illuminated on both sides to increase specific illuminated area 
$\left(a_{\text {light }}=18.2 \mathrm{~m}^{-1}\right)$. The culture is pneumatically agitated. To allow steam sterilization, the PBR is made of 316L-grade stainless steel and runs in continuous culture mode with full regulation of growth parameters such as $\mathrm{pH}$ and temperature. This technology has proven especially suitable for cases where biomass production necessitated well-controlled conditions. An example of its use for optimized lipid production can be found in Pruvost et al (2011b). This technology is currently used in the AlgoSolis R\&D facility to continuously produce microalgal biomass of constant quality (axenic conditions) so as to inoculate large outdoor culture systems (such as enclosed raceways, see next).

\subsection{Solar Technologies}

\subsubsection{Surface and Volumetrically Lighted Systems}

Light can be supplied in two general ways: by directly lighting the culture system or by distributing light sources inside the culture volume. Next, there are either surface-illuminated systems or volumetrically illuminated systems. Most cultivation systems fall in the simpler surface-illuminated category (Carvalho et al, 2006; Morweiser et al, 2010; Richmond, 2004a; Ugwu et al, 2008). As with any other solar process, various positioning options have been considered, including systems positioned horizontally (Acién Fernández et al, 2001; Molina et al, 2001; Oswald, 1988), vertically (Chini Zittelli et al, 2000, 2006; Pulz, 2001), and even tilted (Doucha and Livansky, 2006; Lee and Low, 1991; Richmond and Cheng-Wu, 2001). However, maximizing the incident solar radiation flux is no easy task. For a start, it depends on longitude and latitude of system location and on the day of the year. For example, horizontal systems are best suited for locations close to the equator (latitude 0 degree). For higher latitudes, it is necessary to tilt the exposed system surface to maximize the amount of light collected. Roughly speaking, the optimum inclination angle with respect to the Earth's surface to maximize light capture over the year on a fixed PBR corresponds to the latitude of the PBR location (Duffie and Beckman, 2006; Hu et al, 1996; Pruvost et al, 2012; Richmond and Cheng-Wu, 2001). Inclination angle can also be adjusted as a function of time to optimize light capture. Flat panels equipped with sun-tracking systems were tested by Hindersin et al (2013), and the method not only maximized light capture during the day but also prevented excessive incident irradiation on the systems around noon by temporarily setting the illuminated surface of the PBR perpendicular to the sun's collimated irradiation. 
Volumetrically illuminated systems require more complex technologies than surface-illuminated systems, but they do enable optimization of light delivery and use in the culture. First, inserting light sources in the volume of the culture guarantees maximal use of the collected or emitted photons. Second, and more interestingly, internal lighting allows light to be "diluted." As discussed earlier, increasing PFD leads to higher volumetric productivity but also a progressive decrease in conversion yield due to photosynthesis saturation. By diluting the light incident on the system's surface into the volume of the culture, a larger yield can be maintained. This is of particular interest in outdoor PBRs exposed to sunlight. In this case, the solar radiation incident on a given surface is collected using a parabolic solar collector for example, and is then delivered to the culture in a controlled manner, using optical fibers (Cornet, 2010; Csogör et al, 2001) or light guides (Pilon et al, 2011) for example. The characteristically high PFD of solar conditions means surface productivity can be increased. Note that the optical connection between the light collection device and the light delivery system needs to be carefully designed as it can be a source of major optical losses. Furthermore, light dilution can be combined with a solar tracking system, offering an added possibility of optimization by maximizing light intercepted as the sun travels in the sky (Hindersin et al, 2013). A full description of such a principle has been described by Cornet (2010) with a volumetrically lightened PBR based on the "DiCoFluV" concept (more details are given in next sections). Volumetrically illuminated PBRs hold great promise, but there are still rare few examples in the literature (Cornet, 2010; Csogör et al, 2001; Hsieh and Wu, 2009; Ogbonna et al, 1996; Zijffers et al, 2008), mainly due to the technological complexity involved and the difficulty scaling up PBR systems to large surface areas.

\subsubsection{Examples of Surface-Lightened PBRs}

\subsubsection{Covered Raceway}

The raceway is a horizontal planar cultivation system that has gained currency as a mature cultivation system (Becker, 1994; Oswald, 1962, 1988). Raceway technologies are effectively easy to scale-up and cheap to build. Different materials can be used, from clay to PVC (Ben-Amotz, 2008). Over the last few years, optimizations have been proposed, such as using computational fluid dynamics to optimize mixing with a paddlewheel design (Chiaramonti et al, 2013; Hadiyanto et al, 2013; Hreiz et al, 2014; Liffman et al, 2013), to maximize biomass productivity and light 
distribution in the culture volume, and to reduce power consumption (Lundquist et al, 2010).

Like for any open system, raceway technologies are well adapted to producing extremophile microalgae like the cyanobacteria $A$. platensis which is able to grow under high $\mathrm{pH}$ and high-temperature conditions (reducing the risk of bacterial contaminations). On the other hand, if the goal of the system is to produce sensitive microorganisms, then a closed system is needed. AlgoSource recently developed an enclosed raceway (Fig. 2). Covering a raceway system allows to control internal growth conditions for better productivity. It also enables control of the gas phase, such as reducing $\mathrm{CO}_{2}$ desorption, making this technology suitable for flue gas treatment applications. An covered raceway technology was recently implemented at AlgoSolis R\&D facility for this purpose.

\subsubsection{AlgoFilm Technologies}

Fig. 1 illustrates the utility of increasing both specific illuminated surface (or decreasing the culture depth, ie, $a_{\text {light }}=S_{\text {light }} / V_{\mathrm{R}}=1 / L$ for a flat panel) and PFD to increase volumetric productivity (or biomass concentration, the two being linked). This introduces the basic concepts of PBR intensification. More specifically, the interest of working in a thin film $\left(a_{\text {light }}>100 \mathrm{~m}^{-1}\right.$, $L<0.01 \mathrm{~m})$ is clearly demonstrated here: compared to usual geometries ( $a_{\text {light }}$ around $20 \mathrm{~m}^{-1}$ for a PBR of depth $0.05 \mathrm{~m}, 0.3 \mathrm{~m}^{-1}$ for raceway of depth $0.3 \mathrm{~m}$ ), two orders of magnitude can be gained on volumetric productivity, making it possible to work in high-cell-density culture $\left(C_{X}>10 \mathrm{~kg} \mathrm{~m}^{-3}\right)$ leading to the advent of high volumetric productivity PBR (HVP-PBR). Note also that increasing the PFD will lead to a further increase in biomass productivity (but with a decrease in thermodynamic yield of photosynthetic conversion, as previously discussed). As surface productivity is independent of specific illuminated surface (Eq. 2), a specific feature of PBR technology is the possibility of drastically increasing volumetric productivity while maintaining surface productivity. This was the basic statement behind the design of AlgoFilm(C) technology (Fig. 2) which aims to propose very high volumetric productivity (HVP-PBR) at the current performance ceiling while keeping the maximal conversion of incoming light permitted by the direct illumination principle (surface-lightened system, without light dilution).

The direct advantage of intensifying volumetric productivity is that it reduces the system size needed to achieve a given production requirement. In the general framework of a global industrial exploitation, energy 
consumption in several processes is directly linked to culture volume (pumping, mixing, temperature control, harvesting, etc.). Increasing volumetric productivity can thus drastically reduce energy needs for a given operation. This holds primary relevance in biofuel production for example, where both surface and volumetric productivities can be increased by appropriately engineering PBRs. For example, the prototype presented in Fig. 2 has a depth of around $0.002 \mathrm{~m}$ which corresponds to $a_{\text {light }}=500 \mathrm{~m}^{-1}$ (or $2 \mathrm{~L}$ of culture per $\mathrm{m}^{3}$ ). For a given PFD of $200 \mu \mathrm{mol}_{h \nu} \mathrm{m}^{-2} \mathrm{~s}^{-1}$ (corresponding roughly to the yearly averaged value of irradiation in Paris, France), a volumetric productivity $P_{\mathrm{V}}$ around $3.3 \mathrm{~kg} \mathrm{~m}^{-3}$ day ${ }^{-1}$ could be obtained with a surface productivity of $14 \mathrm{~g} \mathrm{~m}^{-2}$ day $^{-1}$. A PBR of higher depth could provide the same maximal surface productivity but with substantial volumetric productivity $\left(0.04 \mathrm{~kg} \mathrm{~m}^{-3}\right.$ day $^{-1}$ for a PBR of $0.15 \mathrm{~m}$ depth, ie, $a_{\text {light }}=0.07 \mathrm{~m}^{-1}$ or $150 \mathrm{~L}$ of culture per $\mathrm{m}^{3}$ ). Note that the AlgoSolis $\mathrm{R} \& \mathrm{D}$ facility recently integrated the AlgoFilm technology as a platform for research projects in microalgal liquid biofuel production (biodiesel, biokerosene).

\subsubsection{Example of a Volume-Lightened PBR}

\subsubsection{Introduction}

Internal illumination is often discussed as a way to improve the productivity of PBRs. However, although the concept appears simple, the design of an efficient system is far from trivial. An inappropriate design can lead to lower productivities than easier-to-build surface-lightened systems. The optical device used for light collection and its transmission to the culture is a critical factor. Even with an optimized optical device, the arrangement and sizing of the light guides used to diffuse light to the culture have to respect some keys parameters, which were identified and fixed by Cornet (2010). An example is given here with cylindrical light guides.

The main parameters in this case are distance between light guides $d_{\mathrm{i}}$, light-guide diameter $d_{\mathrm{s}}$, and volume fraction of the light guides inside the total reactor volume $\varepsilon$. For example, Cornet (2010) defined an optimal spatial distribution $\varepsilon_{\mathrm{opt}}=0.2267$ for cylindrical structures (see later). In addition, as ideal transmission cannot be obtained from optical devices, two energetic transmission yields have to be considered. The first concerns the transmission efficiency from the light-collecting surface to all inlet sections of the light guides $\eta_{0}$, while the second depends on how efficiently the light guides transport and deliver light from the inlet section to the lateral 
surface $\left(\eta_{1}\right)$. In these conditions, the light flux delivered to the culture $\left(q_{2}\right)$ is defined by Eq. (17):

$$
q_{2}=\eta_{0} \eta_{1} q \frac{S_{0}}{\sum S_{2}}
$$

where $S_{0}$ is the light-collecting surface and $\sum S_{2}$ is the outlet surface of light guides.

Once the light flux received by the culture is known, theoretical maximal performances of volume-lightened PBRs can be determined, using Eq. (2) presented earlier (using the light flux $q_{2}$ effectively received by the culture instead of the collected light flux, q). An example given here to emphasize the difficulty of designing an efficient technology is for A. platensis (Cornet and Dussap, 2009) with irradiation conditions obtained in Paris, France (year-averaged PFD). Some favorable assumptions were also retained:

- Ideal transmission efficiencies $\left(\eta_{0}=\eta_{1}=1\right.$, which means light transmission from light source to the culture is equal to 1). In practice, any optical device will introduce a loss of transmitted light, and so the collecting surface $S_{\mathrm{o}}$ has to be increased accordingly.

- Both direct and diffuse components of the solar radiation are transmitted into the culture volume $\left(q_{\text {sunlight }}=q_{/ /}+q_{\cap}\right)$.

- Optimal spatial distribution $\varepsilon_{\text {opt }}$ of light guides in the culture volume.

- No biomass loss during the night.

To illustrate the impact of the light-guide characteristics, an example is given here for a system having a light-collecting surface $S_{0}$ equal to the total footprint surface of the cultivation system. Three values were fixed for the diameter and height of the light guides, ie, 0.5, 1, $2 \mathrm{~m}$ and 3, 7,10 m, respectively. In these conditions, surface productivities obtained from Eq. (2) ranged from 28 to $48 \mathrm{t}$ /(ha year), while volumetric productivities ranged from 0.5 to $1.8 \mathrm{~kg} /\left(\mathrm{m}^{3}\right.$ year). Note that the maximal volumetric and surface productivities are not obtained in the same geometric configurations, which illustrates the difficulty of co-optimizing both the surface and volumetric productivities of volume-lightened PBR. This is roughly explained by the positive effect of the light dilution principle on surface productivity, which conversely decreases volumetric productivity (Fig. 1). The maximal surface productivity reached in volume-lightened PBRs is almost twofold higher than in surfacelightened PBRs (enclosed raceways and AlgoFilm(C) technologies). Around $25 \mathrm{t}$ / (ha year) would be achieved in surface-lightened PBRs using the same simulation conditions. However, the maximal volumetric productivity is 
one order of magnitude higher in enclosed raceways (depth of $15 \mathrm{~cm}$, $P_{\mathrm{V}}=17 \mathrm{~kg} /\left(\mathrm{m}^{3}\right.$ year $\left.)\right)$ and almost three orders of magnitude higher using the AlgoFilm technology (mean depth of $2 \mathrm{~mm}, P_{\mathrm{V}}=1200 \mathrm{~kg} /\left(\mathrm{m}^{3}\right.$ year)).

Various designs can be easily simulated based on engineering equations (Eq. 2). Solar concentration devices can be excluded to keep the technology simple. In practice, this can be achieved by simply immersing optical devices for light dilution in the culture volume, in which case the collecting surface will then be equal to the total footprint surface multiplied by $\varepsilon_{\text {opt }}$. The surface productivities of volume-lightened PBRs then range from 9 to $12 \mathrm{t}$ /(ha year), while volumetric productivities range from 0.15 to $0.50 \mathrm{~kg} /\left(\mathrm{m}^{3}\right.$ year $)$. Light dilution and the absence of solar concentration then mean that the PFD $\left(q_{2}\right)$ received by photosynthetic microorganisms is very low, close to the compensation point of microalgae $\left(A_{\mathrm{C}}\right.$ in the range of $\left.1-3 \mu \mathrm{mol}_{h \nu} \mathrm{g}^{-1} \mathrm{~s}^{-1}\right)$, which leads to very low biomass concentration and volumetric productivity. This clearly demonstrates that volume-lightened PBRs must integrate solar collectors to make the technology viable and efficient in practice. This is the concept of DiCoFluV, which is presented in next section.

\subsubsection{The DiCoFluV PBR}

The DiCoFluV concept (Cornet, 2010) is based on internal volumetric illumination of the culture medium with the optimized light dilution principle. To compensate for the decrease in volumetric productivity due to light dilution, light guides are arranged to provide a very high value of specific illuminated surface $\left(a_{\text {light }}>300 \mathrm{~m}^{-1}\right)$ obtained from the use of thin optical fibers with lateral diffusion of light (diameter typically of few millimeters). The high internal illuminating surface then obtained makes it necessary to introduce a preliminary stage of solar concentration to keep sufficient light entering the culture system. By applying engineering rules for optimal light dilution, this principle enables engineers to work with classical volume bioreactor technologies and to operate very close to the thermodynamic optimum for the solar-to-biomass conversion process, using low incident light fluxes by dilution of the actual full outdoor sunlight.

The development of the corresponding technology requires several stages. First, the conception of the layout for the optical fibers with lateral diffusion of light used inside the culture volume has to be optimized (providing light and diluting the incident solar flux captured outdoor with a high illuminated specific area). This can be achieved by using the constructal approach (leading to the $\varepsilon_{\text {opt }}$ value given in the previous section; Bejan, 
2000; Bejan and Lorente, 2012) or, in the future, by analyzing the geometric sensitivities provided by an integral Monte Carlo formulation of the kinetic coupling with radiative transfer (Dauchet et al, 2013). The concept also imposes working with a low PFD at the surface of the fibers to achieve high thermodynamic efficiency (around 15\% in the PAR). This requires models of light transfer for simple one-dimensional (Cornet, 2010) or complex three-dimensional PBR geometries (Dauchet et al, 2013; Lee et al, 2014). Second, the optimum solar capture area needs to be determined. As explained earlier, this makes it necessary to consider the transmission efficiencies of optical devices used for solar concentration and light transport in light guides up to delivery to the culture, but also to use kinetic models coupling the local light absorption rate $A$ with biomass growth rates to predict the productivities achieved by the PBR as a function of irradiation conditions encountered over a period of exploitation.

This approach was recently adopted to build a DiCoFluV PBR with a total volume of $30 \mathrm{~L}$ and a capture surface using 25 Fresnel lenses (Fig. 2). The optimal light dilution factor of the incident PFD (full sunlight) was found to be relatively constant for any location on Earth. Nevertheless, the concept was clearly demonstrated as more interesting in locations with strong direct illumination. Relatively good volumetric biomass productivities are made possible by the large illuminated surface $a_{\text {light }}$ of roughly $350 \mathrm{~m}^{2} \mathrm{~m}^{-3}$ compensating for the low incident diluted PFD, ensuring high thermodynamic efficiency of solar energy conversion, ie, a lower footprint for this technology. Note that this technology is mainly conceived as an optimal surface biomass productivity concept capable of a fivefold increase in surface productivity (by unit footprint) in solar conditions compared to conventional direct illumination systems (considering losses in the light transmission chain). This corresponds to the maximum thermodynamic efficiency of photosynthesis. Actual system performance depends on the optical efficiency of the capture/concentration/filtration/distribution of light inside the culture vessel. On the demonstrator represented in Fig. 2, transmission efficiency reaches $30 \%$ and can probably be further increased to $50 \%$. Another important advantage of this technology is that the complete spectrum of the sun can be used postconcentration by splitting visible and infrared radiation and converting the infrared to provide the necessary mechanical work to the PBR (pumps, mixing, and so on). This is a crucial point that is generally omitted in most PBR efficiency calculations. With this kind of technology, it could be possible to provide high-value biomass at a thermodynamic efficiency reaching 15\% (defined on the whole incident 
solar spectrum), ie, with the same efficiency as current industrial photovoltaic devices producing only electricity.

\section{CONCLUSION}

This chapter discussed the parameters to consider when designing and operating microalgal cultivation systems and how a robust and rational engineering approach can support optimal system design and operation. Indepth and long-term modeling efforts have produced engineering rules and formulae to design, optimize, and control PBRs in a predictive and rational way. This was illustrated here by giving examples of recent published PBR developments for both artificial light sources and sunlight and for various purposes from lab-scale fundamental research to industrial exploitation. It was shown that factoring practical and economic constraints of the final application into the engineering phase culminates in very different technologies despite sharing the same rational engineering tools at the outset. This emphasizes how microalgal cultivation systems, unlike more classical bioprocesses for heterotrophic growth (ie, yeast, bacteria, etc.) that can work with stand-geometry mixing tanks, have no standard geometry to work to, mainly because light supply has such a big influence on process performances that various technologies have emerged in a battle to maximize light use. However, with appropriate consideration of all the constraints, as illustrated here, it is possible to set a rational design of effective technologies, which is obviously of primary interest for microalgae-based industries.

\section{ACKNOWLEDGMENTS}

This work was supported by several projects, and especially by the French National Research Agency within the framework of the DIESALG (ANR-12-BIME-0001-02) and BIOSOLIS projects. This work is also connected to $\mathrm{R} \& \mathrm{D}$ activities led at the AlgoSolis R\&D facility (www.algosolis.com).

\section{REFERENCES}

Acién Fernández FG, Sevilla JM, Sánchez Pérez JA, Molina Grima E, Chisti Y: Airlift-driven external-loop tubular photobioreactors for outdoor production of microalgae: assessment of design and performance, Chem Eng Sci 56(8):2721-2732, 2001.

Acién FG, Fernández JM, Magan JJ, Molina E: Production cost of a real microalgae production plant and strategies to reduce it, Biotechnol Adv 30(6):1344-1353, 2012.

Aiba S: Growth kinetics of photosynthetic microorganisms, Adv Biochem Eng Biotechnol 23:85-156, 1982.

Becker EW: Microalgae: biotechnology and microbiology, vol 10, Cambridge, 1994, Cambridge University Press.

Bejan A: Shape and structure, from engineering to nature, Cambridge, 2000, Cambridge University Press. 
Bejan A, Lorente S: The physics of spreading ideas, Int J Heat Mass Transf 55(4):802-807, 2012.

Ben-Amotz A: Large-scale open algae ponds, NREL-AFOSR joint workshop on algal oil for jet fuel production, 2008.

Berberoglu H, Pilon L, Melis A: Radiation characteristics of Chlamydomonas reinhardtii CC125 and its truncated chlorophyll antenna transformants tla1, tlaX and tla1-CW +, Int J Hydrogen Energy 33(22):6467-6483, 2008.

Bergmann P, Ripplinger P, Beyer L, Trösch W: Disposable flat panel airlift photobioreactors, Chem Ing Tech 85(1-2):202-205, 2013.

Bitog JP, Lee IB, Lee CG, et al: Application of computational fluid dynamics for modeling and designing photobioreactors for microalgae production: a review, Comput Electron Agr 76(2):131-147, 2011.

Borowitzka MA: Microalgae for aquaculture: opportunities and constraints, J Appl Phycol 9:393-401, 1997.

Borowitzka MA: Commercial production of microalgae: ponds, tanks, and fermenters, Prog Ind Microbiol 35:313-321, 1999.

Carvalho AP, Meireles LA, Malcata FX: Microalgal reactors: a review of enclosed system designs and performances, Biotechnol Prog 22:1490-1506, 2006.

Carvalho AP, Silva SO, Baptista JM, Malcata FX: Light requirements in microalgal photobioreactors: an overview of biophotonic aspects, Appl Microbiol Biotechnol 89(5):1275-1288, 2011.

Cassano AE, Martin CA, Brandi RJ, Alfano OM: Photoreactor analysis and design: fundamentals and applications, Ind Eng Chem Res 34(7):2155-2201, 1995.

Chen C-Y, Lee C-M, Chang J-S: Feasibility study on bioreactor strategies for enhanced photohydrogen production from Rhodopseudomonas palustris WP3-5 using optical-fiberassisted illumination systems, Int J Hydrogen Energy 31:2345-2355, 2006.

Chiaramonti D, Prussi M, Casini D, et al: Review of energy balance in raceway ponds for microalgae cultivation: re-thinking a traditional system is possible, Appl Energy 102:101-111, 2013.

Chini Zittelli GC, Pastorelli R, Tredici MR: A modular flat panel photobioreactor (MFPP) for indoor mass cultivation of Nannochloropsis sp. under artificial illumination, $J$ Appl Phycol 12(3-5):521-526, 2000.

Chini Zittelli G, Rodolfi L, Biondi N, Tredici MR: Productivity and photosynthetic efficiency of outdoor cultures of Tetraselmis suecica in annular columns, Aquaculture 261(3):932-943, 2006.

Chisti MY: Airlift bioreactors, ed 1, London, England and New York, USA, 1989, Elsevier Applied Science.

Cogne G, Rugen M, Bockmayr A, et al: A model-based method for investigating bioenergetic processes in autotrophically growing eukaryotic microalgae: application to the green alga Chlamydomonas reinhardtii, Biotechnol Prog 27(3):631-640, 2011.

Cornet JF: Etude cinétique et énergétique d'un photobioréacteur. Etablissement d'un modèle structuré. Applications à un écosystème clos artificiel, Orsay, 1992, Université Paris XI.

Cornet J-F: Calculation of optimal design and ideal productivities of volumetrically lightened photobioreactors using the constructal approach, Chem Eng Sci 65(2): 985-998, 2010.

Cornet JF, Albiol J: Modeling photoheterotrophic growth kinetics of Rhodospirillum rubrum in rectangular photobioreactors, Biotechnol Prog 16:199-207, 2000.

Cornet JF, Dussap CG: A simple and reliable formula for assessment of maximum volumetric productivities in photobioreactors, Biotechnol Prog 25:424-435, 2009.

Cornet JF, Dussap CG, Cluzel P, Dubertret G: A structured model for simulation of cultures of the cyanobacterium Spirulina platensis in photobioreactors. 1. Coupling between light transfer and growth kinetics, Biotechnol Bioeng 40(7):817-825, 1992 a. 
Cornet JF, Dussap CG, Cluzel P, Dubertret G: A structured model for simulation of cultures of the cyanobacterium Spirulina platensis in photobioreactors. 2. Identification of kinetic parameters under light and mineral limitations, Biotechnol Bioeng 40(7):826-834, 1992b.

Cornet JF, Dussap CG, Gros JB: Conversion of radiant light energy in photobioreactors, AIChE J 40(6):1055-1066, 1994.

Cornet JF, Dussap CG, Gros JB: A simplified monodimensional approach for modeling coupling between radiant light transfer and growth kinetics in photobioreactors, Chem Eng Sci 50(9):1489-1500, 1995.

Cornet JF, Dussap CG, Gros JB: Kinetics and energetics of photosynthetic micro-organisms in photobioreactors: application to Spirulina growth, Adv Biochem Eng Biotechnol 59:155-224, 1998.

Cornet JF, Favier L, Dussap CG: Modeling stability of photoheterotrophic continuous cultures in photobioreactors, Biotechnol Prog 19(4):1216-1227, 2003.

Cournac L, Musa F, Bernard L, Guedeney G, Vignais P, Peltier G: Limiting steps of hydrogen production in Chlamydomonas reinhardtii and Synechocystis PCC 6803 as analysed by light-induced gas exchange transients, Int J Hydrogen Energy 27:1229-1237, 2002.

Csogör Z, Herrenbauer M, Schmidt K, Posten C: Light distribution in a novel photobioreactor-modelling for optimization, J Appl Phycol 13:325-333, 2001.

Dauchet J: Analyse radiative des photobioreacteurs, PhD thesis, Clermont-Ferrand, France, 2012, Universite Blaise Pascal ( ${ }^{\circ}$ ordre 2304-in French).

Dauchet J, Blanco S, Cornet JF, El Hafi M, Eymet V, Fournier R: The practice of recent radiative transfer Monte Carlo advances and its contribution to the field of microorganisms cultivation in photobioreactors, J Quant Spectrosc Radiat Transf 128:52-59, 2013.

Dauchet J, Blanco S, Cornet J-F, Fournier R: Calculation of the radiative properties of photosynthetic microorganisms, J Quant Spectrosc Radiat Transf 161:60-84, 2015.

Degrenne B, Cogne G, Pruvost J, Legrand J: Role of acetate and light attenuation in hydrogen production by Chlamydomonas reinhardtii, J Biotechnol 136:S559, 2008.

Degrenne B, Pruvost J, Christophe G, Cornet JF, Cogne G, Legrand J: Investigation of the combined effects of acetate and photobioreactor illuminated fraction in the induction of anoxia for hydrogen production by Chlamydomonas reinhardtii, Int J Hydrogen Energy 35(19):10741-10749, 2010.

Degrenne B, Pruvost J, Legrand J: Effect of prolonged hypoxia in autotrophic conditions in the hydrogen production by the green microalga Chlamydomonas reinhardtii in photobioreactor, Bioresour Technol 102:1035-1043, 2011a.

Degrenne B, Pruvost J, Titica M, Takache H, Legrand J: Kinetic modeling of light limitation and sulfur deprivation effects in the induction of hydrogen production with Chlamydomonas reinhardtii. Part II: definition of model-based protocols and experimental validation, Biotechnol Bioeng 108(10):2288-2299, 2011 b.

Doucha J, Livansky K: Productivity, CO2/O2 exchange and hydraulics in outdoor open high density microalgal (Chlorella sp.) photobioreactors operated in a Middle and Southern European climate, J Appl Phycol 18:811-826, 2006.

Duffie JA, Beckman WA: Solar engineering of thermal processes, ed 3, New York, 2006, John Wiley \& Sons.

Farges B, Laroche C, Cornet J-F, Dussap C-G: Spectral kinetic modeling and long-term behavior assessment of Arthrospira platensis growth in photobioreactor under red (620 nm) light illumination, Biotechnol Prog 25:151-162, 2009.

Fouchard S, Hemschemeier A, Caruana A, et al: Autotrophic and mixotrophic hydrogen photoproduction in sulfur-deprived Chlamydomonas cells, Appl Environ Microbiol 71(10):6199-6205, 2005.

Fouchard S, Pruvost J, Degrenne B, Legrand J: Investigation of H2 production using the green microalga Chlamydomonas reinhardtii in a fully controlled photobioreactor fitted with on-line gas analysis, Int J Hydrogen Energy 33(13):3302-3310, 2008. 
Fouchard S, Pruvost J, Degrenne B, Titica M, Legrand J: Kinetic modeling of light limitation and sulphur deprivation effects in the induction of hydrogen production with Chlamydomonas reinhardtii Part I: model description and parameters determination, Biotechnol Bioeng 102(1):132-147, 2009.

Goetz V, Le Borgne F, Pruvost J, Plantard G, Legrand J: A generic temperature model for solar photobioreactors, Chem Eng J 175:443-449, 2011.

Gonzalez de la Vara L, Gomez-Lojero C: Participation of plastoquinone, cytochrome c553 and ferredoxin-NADP + oxido reductase in both photosynthesis and respiration in Spirulina maxima, Photosynth Res 8:65-78, 1986.

Grima ME, Sevilla FJM, Perez JAS, Camacho FG: A study on simultaneous photolimitation and photoinhibition in dense microalgal cultures taking into account incident and averaged irradiances, J Biotechnol 45:59-69, 1996.

Grima ME, Fernandez AFG, Camacho GF, Chisti Y: Photobioreactors: light regime, mass transfer, and scaleup, J Biotechnol 70:231-247, 1999.

Grobbelaar JU: Factors governing algal growth in photobioreactors: the "open" versus “closed" debate, J Appl Phycol 21(5):489-492, 2008.

Hadiyanto H, Elmore S, Van Gerven T, Stankiewicz A: Hydrodynamic evaluations in high rate algae pond (HRAP) design, Chem Eng J 217:231-239, 2013.

Hindersin S: Photosynthetic efficiency of microalgae and optimization of biomass production in photobioreactors, Hamburg, 2013, Universitaät Hamburg, Dissertation.

Hindersin S, Leupold M, Kerner M, Hanelt D: Irradiance optimization of outdoor microalgal cultures using solar tracked photobioreactors, Bioprocess Biosyst Eng 36(3):345-355, 2013.

Hindersin S, Leupold M, Kerner M, Hanelt D: Key parameters for outdoor biomass production of Scenedesmus obliquus in solar tracked photobioreactors, J Appl Phycol 26:2315-2325, 2014.

Hreiz R, Sialve B, Morchain J, Escudié R, Steyer J-P, Guiraud P: Experimental and numerical investigation of hydrodynamics in raceway reactors used for algaculture, Chem EngJ 250:230-239, 2014.

Hsieh CH, Wu WT: A novel photobioreactor with transparent rectangular chambers for cultivation of microalgae, Biochem Eng J 46(3):300-305, 2009.

Hu Q, Guterman H, Richmond A: A flat inclined modular photobioreactor for outdoor mass cultivation of photoautotrophs, Biotechnol Bioeng 51(1):51-60, 1996.

Ifrim GA, Titica M, Cogne G, Boillereaux L, Legrand J, Caraman S: Dynamic pH model for autotrophic growth of microalgae in photobioreactor: a tool for monitoring and control purposes, AIChE J 60(2):585-599, 2014.

James SC, Janardhanam V, Hanson DT, Mock T: Simulating pH effects in an algal-growth hydrodynamics model, J Phycol 49(3):608-615, 2013.

Janssen M, Janssen MGJ, De Winter M, et al: Efficiency of light utilization of Chlamydomonas reinhardtii under medium-duration light/dark cycles, J Biotechnol 78:123-137, 2000.

Janssen M, Tramper J, Mur LR, Wijffels RH: Enclosed outdoor photobioreactors: light regime, photosynthetic efficiency, scale-up, and future prospects, Biotechnol Bioeng 81(2):193-210, 2003.

Kandilian R, Lee E, Pilon L: Radiation and optical properties of Nannochloropsis oculata grown under different irradiances and spectra, Bioresour Technol 137:63-73, 2013.

Kandilian R, Pruvost J, Legrand J, Pilon L: Influence of light absorption rate by Nannochloropsis oculata on triglyceride production during nitrogen starvation, Bioresour Technol 163:308-319, 2014.

Le Borgne F: Développement d'un photobioréacteur solaire intensifié en vue de la production à grande échelle de biomasse microalgale, Saint-Nazaire, 2011, Université de Nantes.

Le Borgne F, Pruvost J: Investigation and modeling of biomass decay rate in the dark and its potential influence on net productivity of solar photobioreactors for microalga 
Chlamydomonas reinhardtii and cyanobacterium Arthrospira platensis, Bioresour Technol 138:271-276, 2013.

Lee Y-K, Low C-S: Effect of photobioreactor inclination on the biomass productivity of an outdoor algal culture, Biotechnol Bioeng 38(9):995-1000, 1991.

Lee E, Pruvost J, He X, Munipalli R, Pilon L: Design tool and guidelines for outdoor photobioreactors, Chem Eng Sci 106:18-29, 2014.

Le Gouic B: Analyse et optimisation de l'apport de carbone en photobioréacteur, $\mathrm{PhD}$ thesis, 2013, University of Nantes.

Lehr F, Posten C: Closed photo-bioreactors as tools for biofuel production, Curr Opin Biotechnol 20(3):280-285, 2009.

Liffman K, Paterson DA, Liovic P, Bandopadhayay P: Comparing the energy efficiency of different high rate algal raceway pond designs using computational fluid dynamics, Chem Eng Res Des 91(2):221-226, 2013.

Loubiere K, Olivo E, Bougaran G, Pruvost J, Robert R, Legrand J: A new photobioreactor for continuous microalgal production in hatcheries based on external-loop airlift and swirling flow, Biotechnol Bioeng 102(1):132-147, 2009.

Loubiere K, Pruvost J, Aloui F, Legrand J: Investigations in an external-loop airlift photobioreactor with annular light chambers and swirling flow, Chem Eng Res Des 89(2):164-171, 2011.

Lundquist TJ, Woertz IC, Quinn NWT, Benemann JR: A realistic technology and engineering assessment of algae biofuel production, Berkeley, California, 2010, Energy Biosciences Institute.

Martzolff A, Cahoreau E, Cogne G, et al: Photobioreactor design for isotopic non-stationary 13C-metabolic flux analysis (INST 13C-MFA) under photoautotrophic conditions, Biotechnol Bioeng 109(12):3030-3040, 2012.

Molina E, Fernández J, Acién FG, Chisti Y: Tubular photobioreactor design for algal cultures, J Biotechnol 92(2):113-131, 2001.

Morweiser M, Kruse O, Hankamer B, Posten C: Developments and perspectives of photobioreactors for biofuel production, Appl Microbiol Biotechnol 87(4):1291-1301, 2010.

Muller-Feuga A, Le Guédes R, Pruvost J: Benefits and limitations of modeling for optimization of Porphyridium cruentum cultures in an annular photobioreactor, $J$ Biotechnol 103(2):153-163, 2003a.

Muller-Feuga A, Pruvost J, Le Guedes R, Le Dean L, Legentilhomme P, Legrand J: Swirling flow implementation in a photobioreactor for batch and continuous cultures of Porphyridium cruentum, Biotechnol Bioeng 84(5):544-551, $2003 \mathrm{~b}$.

Myers J, Kratz WA: Relation between pigment content and photosynthetic characteristics in a blue-green algae, J Gen Physiol 39(1):11-22, 1955.

Ogbonna JC, Tanaka H: Night biomass loss and changes in biochemical composition of cells during light/dark cyclic culture of Chlorella pyrenoidosa, J Ferment Bioeng 82(6):558-564, 1996.

Ogbonna JC, Yada H, Masui H, Tanaka H: A novel internally illuminated stirred tank photobioreactor for large-scale cultivation of photosynthetic cells, J Ferment Bioeng 82(1):61-67, 1996.

Oswald WJ: The coming industry of controlled photosynthesis, Am J Public Health Nations Health 52(2):235-242, 1962.

Oswald WJ: Large-scale algal culture systems (engineering aspects). In Borowitska M, editor: Microalgal biotechnology, Cambridge, 1988, Cambridge University Press, pp 357-394.

Peltier G, Thibault P: Uptake in the light in Chlamydomonas. Evidence for persistent mitochondrial respiration, Plant Physiol 79:225-230, 1985.

Perner-Nochta I, Posten C: Simulations of light intensity variation in photobioreactors, J Biotechnol 131(3):276-285, 2007. 
Pilon L, Berberoglu H, Kandilian R: Radiation transfer in photobiological carbon dioxide fixation and fuel production by microalgae, $J$ Quant Spectrosc Radiat Transf 112(17):2639-2660, 2011.

Pottier L, Pruvost J, Deremetz J, Cornet JF, Legrand J, Dussap CG: A fully predictive model for one-dimensional light attenuation by Chlamydomonas reinhardtii in a torus photobioreactor, Biotechnol Bioeng 91(5):569-582, 2005.

Pruvost J: Cultivation of algae in photobioreactors for biodiesel production. In Pandey A, Larroche C, Ricke SC, Dussap CG, editors: Biofuels: alternative feedstocks and conversion processes, USA, 2011, Academic Press, pp 439-464.

Pruvost J, Cornet JF: Knowledge models for engineering and optimization of photobioreactors. In Posten C, Walter C, editors: Microalgal biotechnology, Berlin/Boston, 2012, Walter De Gruyter GmbH \& Co. KG, pp 181-224.

Pruvost J, Legrand J, Legentilhomme P, Muller-Feuga A: Simulation of microalgae growth in limiting light conditions-flow effect, AIChE J 48:1109-1120, 2002a.

Pruvost J, Legrand J, Legentilhomme P, Muller-Feuga A: Lagrangian trajectory model for turbulent swirling flow in an annular cell. Comparison with RTD measurements, Chem Eng Sci 57(7):1205-1215, 2002b.

Pruvost J, Pottier L, Legrand J: Numerical investigation of hydrodynamic and mixing conditions in a torus photobioreactor, Chem Eng Sci 61(14):4476-4489, 2006.

Pruvost J, Cornet JF, Legrand J: Hydrodynamics influence on light conversion in photobioreactors: an energetically consistent analysis, Chem Eng Sci 63:3679-3694, 2008.

Pruvost J, Van Vooren G, Cogne G, Legrand J: Investigation of biomass and lipids production with Neochloris oleoabundans in photobioreactor, Bioresour Technol 100:5988-5995, 2009.

Pruvost J, Cornet JF, Goetz V, Legrand J: Modeling dynamic functioning of rectangular photobioreactors in solar conditions, AIChE J 57(7):1947-1960, 2011a.

Pruvost J, Van Vooren G, Le Gouic B, Couzinet-Mossion A, Legrand J: Systematic investigation of biomass and lipid productivity by microalgae in photobioreactors for biodiesel application, Bioresour Technol 102:150-158, 2011 b.

Pruvost J, Cornet JF, Goetz V, Legrand J: Theoretical investigation of biomass productivities achievable in solar rectangular photobioreactors for the cyanobacterium Arthrospira platensis, Biotechnol Prog 28(3):699-714, 2012.

Pruvost J, Cornet JF, Le Borgne F, Goetz V, Legrand J: Theoretical investigation of microalgae culture in the light changing conditions of solar photobioreactor production and comparison with cyanobacteria, Algal Res 10:87-99, 2015.

Pruvost J, Le Gouic B, Lepine O, Legrand J, Le Borgne F: Microalgae culture in buildingintegrated photobioreactors: biomass production modelling and energetic analysis, Chem Eng J 284:850-861, 2016.

Pulz O: Photobioreactors: production systems for phototrophic microorganisms, Appl Microbiol Biotechnol 57(3):287-293, 2001.

Quinn J, De Winter L, Bradley T: Microalgae bulk growth model with application to industrial scale systems, Bioresour Technol 102:5083-5092, 2011.

Richmond A: Handbook of microalgal culture: biotechnology and applied phycology, Oxford, UK, 2004a, Blackwell Sciences Ltd.

Richmond A: Principles for attaining maximal microalgal productivity in photobioreactors: an overview, Hydrobiologia 512:33-37, 2004b.

Richmond A, Cheng-Wu Z: Optimization of a flat plate glass reactor for mass production of Nannochloropsis sp. outdoors, J Biotechnol 85(3):259-269, 2001.

Rosello Sastre R, Csögör Z, Perner-Nochta I, Fleck-Schneider P, Posten C: Scale-down of microalgae cultivations in tubular photo-bioreactors-a conceptual approach, $J$ Biotechnol 132(2):127-133, 2007.

Slegers PM, Wijffels RH, Van Straten G, Van Boxtel AJB: Design scenarios for flat panel photobioreactors, J Appl Energy 88:3342-3353, 2011. 
Slegers PM, Lösing MB, Wijffels RH, van Straten G, van Boxtel AJB: Scenario evaluation of open pond microalgae production, Algal Res 2(4):358-368, 2013a.

Slegers PM, van Beveren PJM, Wijffels RH, van Straten G, van Boxtel AJB: Scenario analysis of large scale algae production in tubular photobioreactors, Appl Energy 105:395-406, $2013 b$.

Soulies A, Castelain C, Burghelea TI, Legrand J, Marec H, Pruvost J: Investigation and modeling of the effects of light spectrum and incident angle on the growth of Chlorella vulgaris in photobioreactors, Biotechnol Prog, accepted.

Takache H, Christophe G, Cornet JF, Pruvost J: Experimental and theoretical assessment of maximum productivities for the microalgae Chlamydomonas reinhardtii in two different geometries of photobioreactors, Biotechnol Prog 26(2):431-440, 2010.

Takache H, Pruvost J, Cornet JF: Kinetic modeling of the photosynthetic growth of Chlamydomonas reinhardtii in a photobioreactor, Biotechnol Prog 28(3):681-692, 2012.

Takache H, Pruvost J, Marec H: Investigation of light/dark cycles effects on the photosynthetic growth of Chlamydomonas reinhardtii in conditions representative of photobioreactor cultivation, Algal Res 8:192-204, 2015.

Taleb A, Pruvost J, Legrand J, et al: Development and validation of a screening procedure of microalgae for biodiesel production: application to the genus of marine microalgae Nannochloropsis, Bioresour Technol 177:224-232, 2015.

Torzillo G, Accolla P, Pinzani E, Masojidek J: In situ monitoring of chlorophyll fluorescence to assess the synergistic effect of low temperature and high irradiance stresses in Spirulina cultures grown outdoors in photobioreactors, J Appl Phycol 8(4-5):283-291, 1996.

Ugwu CU, Aoyagia H, Uchiyamaa H: Photobioreactors for mass cultivation of algae, Bioresour Technol 99(10):4021-4028, 2008.

Wilhelm C, Selmar D: Energy dissipation is an essential mechanism to sustain the viability of plants: the physiological limits of improved photosynthesis, J Plant Physiol 168(2):79-87, 2011.

Wu X, Merchuk JC: A model integrating fluid dynamics in photosynthesis and photoinhibition processes, Chem Eng Sci 56:3527-3538, 2001.

Zijffers JW, Janssen M, Tramper J, Wijffels RH: Design process of an area-efficient photobioreactor, Marine Biotechnol 10:404-415, 2008.

Zonneveld C: Photoinhibition as affected by photoacclimation in phytoplankton: a model approach, J Theor Biol 193(1):115-123, 1998. 